\title{
Optimal Control of History-Dependent Evolution Inclusions with Applications to Frictional Contact
}

\author{
Stanisław Migórski ${ }^{1,2}$
}

Received: 8 June 2019 / Accepted: 16 March 2020 / Published online: 30 March 2020

(c) The Author(s) 2020

\begin{abstract}
In this paper, we study a class of subdifferential evolution inclusions involving historydependent operators. First, we improve an existence and uniqueness theorem and prove the continuous dependence result in the weak topologies. Next, we establish the existence of optimal solution to an optimal control problem for the evolution inclusion. Finally, we illustrate the results by an example of an optimal control of a dynamic frictional contact problem in mechanics, whose weak formulation is the evolution variational inequality.
\end{abstract}

Keywords Optimal control · Evolution inclusion • Variational inequality · History-dependent operator · Frictional contact

Mathematics Subject Classification 49A22 - 49J24 · 49J27 · 35K86 - 35L86 - 73T05 · $74 \mathrm{M} 10$

\section{Introduction}

This work studies an optimal control problem for a nonlinear infinite dimensional system, whose dynamics is monitored by an evolution inclusion of the subdifferential type involving history-dependent operators. The evolution inclusion is studied in the framework of an evolution triple of spaces.

Communicated by Felix L. Chernousko.

Stanisław Migórski

stanislaw.migorski@uj.edu.pl

1 College of Applied Mathematics, Chengdu University of Information Technology, Chengdu 610225, Sichuan Province, People's Republic of China

2 Chair of Optimization and Control, Jagiellonian University in Krakow, ul. Lojasiewicza 6, 30348 Kraków, Poland 
The optimal control of evolution problems has been extensively studied in the literature; the results on existence, necessary conditions of optimality, and applications can be found, for instance, in Lions [1], Tröltzsch [2] for differential equations, Ahmed and Teo for distributed parameter systems [3], and Barbu [4] and Tiba [5] for variational inequalities. The optimal control problems for the subdifferential evolution inclusions have been examined in many works; see, e.g., [6-11] and the references therein. In all aforementioned contributions, the subdifferential term has values in a Hilbert space or in the pivot space of an evolution triple.

Our initial motivation to study the subdifferential evolution inclusion comes from contact mechanics, where several models of the contact problems can be formulated as the evolution variational inequalities with history-dependent terms. We refer to [1218] for the related papers, successfully exploiting the notion of the history-dependent operator, and to a recent monograph [19] for a comprehensive study. On the other hand, despite great importance, the optimal control for contact problems in solid mechanics has been studied only recently and mainly for static formulations; see [20-27].

In the present paper, we turn our attention to the Cauchy problem for a class of evolution inclusions considered in the framework of an evolution triple of spaces. The main feature of the inclusion under consideration is that the potential function is convex and finite, and the multivalued term is generated by the subdifferential operator, which takes its values in the dual space. Moreover, such inclusions involve two nonlinear operators supposed to be history dependent. Existence and uniqueness results for such problems have been recently proved under various strong hypotheses involving smallness conditions; see [28-33]. The novelties of our paper are the following. We remove the smallness condition used in the earlier works. Further, for the first time, we examine the continuous dependence of the solution to these inclusions on the second member and initial condition and study the Bolza-type optimal control problem. Finally, we provide an illustration of our abstract results to an optimal control problem for a dynamic model of a viscoelastic contact with friction.

The outline of the paper is as follows. After recalling essential tools in Sects. 2 and 3, we establish the unique solvability of a history-dependent inclusion with the Clarke subdifferential term. Sections 4 and 5 deal with a continuous dependence on the data and an optimal control problem, respectively. Section 6 provides an example of an optimal control problem for a dynamic frictional contact problem, whose weak formulation leads to an evolution variational inequality equivalent to the evolution inclusion. Finally, we note that the continuous dependence result, in the weak topologies, obtained in this paper can be used in the analysis of various optimal control problems for variational inequalities with history-dependent operators.

\section{Notation and Preliminaries}

Let $X$ be a Banach space. Throughout the paper, we denote by $\langle\cdot, \cdot\rangle_{X^{*} \times X}$ the duality pairing between $X$ and its dual $X^{*}$, and by $\|\cdot\|_{X}$ the norm in $X$. When no confusion arises, we often drop the subscripts. A function $\varphi: X \rightarrow \mathbb{R} \cup\{+\infty\}$ is proper, if its effective domain $\operatorname{dom} \varphi=\{x \in X: \varphi(x)<+\infty\} \neq \emptyset$. It is sequentially lower semicontinuous (l.s.c.), if $\varphi(x) \leq \liminf \varphi\left(x_{n}\right)$ for $x_{n} \rightarrow x$ in $X$. Given a convex 
function $\varphi: X \rightarrow \mathbb{R} \cup\{+\infty\}$, an element $x^{*} \in X^{*}$ is called a subgradient of $\varphi$ at $u \in X$, if

$$
\left\langle x^{*}, v-u\right\rangle_{X^{*} \times X} \leq \varphi(v)-\varphi(u) \text { for all } v \in X .
$$

The set of all elements $x^{*} \in X^{*}$, which satisfy (1), is called the (convex) subdifferential of $\varphi$ at $u$ and is denoted by $\partial \varphi(u)$. Next, we recall the notion of the Clarke generalized gradient for a locally Lipschitz function $\psi: X \rightarrow \mathbb{R}$. The generalized gradient of $\psi$ at $u \in X$ is defined by

$$
\partial \psi(u)=\left\{u^{*} \in X^{*}: \psi^{0}(u ; v) \geq\left\langle u^{*}, v\right\rangle_{X^{*} \times X} \text { for all } v \in X\right\},
$$

where the generalized directional derivative of $\psi$ at $u \in X$ in the direction $v \in X$ is given by

$$
\psi^{0}(u ; v)=\limsup _{y \rightarrow u, t \downarrow 0} \frac{\psi(y+t v)-\psi(y)}{t} .
$$

In what follows, the generalized gradient of Clarke for a locally Lipschitz function and the subdifferential of a convex function will be denoted in the same way.

Given a set $S$ in a normed space $E$, we define $\|S\|_{E}=\sup \left\{\|x\|_{E}: x \in S\right\}$. The space of linear and bounded operators from a normed space $E$ to a normed space $F$ is denoted by $\mathcal{L}(E, F)$. It is endowed with the standard operator norm $\|\cdot\|_{\mathcal{L}(E, F)}$. For an operator $L \in \mathcal{L}(E, F)$, we denote its adjoint by $L^{*} \in \mathcal{L}\left(F^{*}, E^{*}\right)$.

An operator $A: X \rightarrow X^{*}$ is said to be demicontinuous, if for all $v \in X$, the map $u \mapsto\langle A u, v\rangle_{X^{*} \times X}$ is continuous, i.e., $A$ is continuous as a functional from $X$ to $X^{*}$ endowed with the weak topology. The Nemytskii (superposition) operator $\mathcal{A}: L^{2}(0, T ; X) \rightarrow L^{2}\left(0, T ; X^{*}\right)$, associated with $\left.A:\right] 0, T\left[\times X \rightarrow X^{*}\right.$, is defined by $(\mathcal{A} v)(t)=A(t, v(t))$ for $v \in L^{2}(0, T ; X)$, a.e. $\left.t \in\right] 0, T$ [. A multivalued operator $A: X \rightrightarrows 2^{X^{*}}$ is called coercive, if either its domain $D(A)=\{u \in X: A u \neq \emptyset\}$ is bounded or $D(A)$ is unbounded and

$$
\lim _{\|u\|_{X} \rightarrow \infty, u \in D(A)} \frac{\inf \left\{\left\langle u^{*}, u\right\rangle_{X^{*} \times X}: u^{*} \in A u\right\}}{\|u\|_{X}}=\infty .
$$

Recall that the spaces $\left(V, H, V^{*}\right)$ form an evolution triple of spaces, if $V$ is a reflexive and separable Banach space, $H$ is a separable Hilbert space, and the embedding $V \subset H$ is dense and continuous. In addition, we assume that $V \subset H$ compactly. In concrete applications, evolution triples are generated by Sobolev spaces. Let $0<T<\infty$ and define $\mathcal{V}=L^{2}(0, T ; V), \mathcal{V}^{*}=L^{2}\left(0, T ; V^{*}\right)$, and $\mathcal{W}=\left\{w \in \mathcal{V}: w^{\prime} \in \mathcal{V}^{*}\right\}$. It follows from standard results, see, e.g., [34, Section 8.4], that the space $\mathcal{W}$ endowed with the graph norm $\|w\|_{\mathcal{W}}=\|w\|_{\mathcal{V}}+\left\|w^{\prime}\right\| \mathcal{V}^{*}$ is a separable, reflexive Banach space, and each element in $\mathcal{W}$, after a modification on a set of null measure, can be identified with a unique continuous function on $[0, T]$ with values in $H$. Further, the embedding $\mathcal{W} \subset C(0, T ; H)$ is continuous, where $C(0, T ; H)$ stands for the space of continuous functions on $[0, T]$ with values in $H$. For more details, we refer to [34-36].

Finally, we recall a fixed point result (see [37, Lemma 7] or [38, Proposition 3.1]), which is a consequence of the Banach contraction principle. 
Lemma 2.1 Let $X$ be a Banach space and $0<T<\infty$. Consider an operator $F: L^{2}(0, T ; X) \rightarrow L^{2}(0, T ; X)$ such that

$$
\left\|\left(F v_{1}\right)(t)-\left(F v_{2}\right)(t)\right\|_{X}^{2} \leq c \int_{0}^{t}\left\|v_{1}(s)-v_{2}(s)\right\|_{X}^{2} \mathrm{~d} s
$$

for all $v_{1}, v_{2} \in L^{2}(0, T ; X)$, a.e. $\left.t \in\right] 0, T[$ with a constant $c>0$. Then, there exists a unique $v^{*} \in L^{2}(0, T ; X)$ such that $F v^{*}=v^{*}$.

\section{History-Dependent Evolution Inclusions}

We begin with the study of existence and uniqueness for an abstract first-order subdifferential evolution inclusion. Our study is a continuation of paper [32], where a class of history-dependent variational inequalities has been investigated. The aim is to provide an improved version of the result in [32, Theorem 5], which actually holds with a relaxed smallness hypothesis.

We study an operator inclusion in the standard functional setting used for evolution problems, which exploits the notion of an evolution triple of spaces $\left(V, H, V^{*}\right)$. We use the notation $\mathcal{V}, \mathcal{V}^{*}$ and $\mathcal{W}$, recalled in the previous section.

Given $A:] 0, T\left[\times V \rightarrow V^{*}, \psi:\right] 0, T[\times V \rightarrow \mathbb{R}, f:] 0, T\left[\rightarrow V^{*}\right.$ and $w_{0} \in V$, we consider the following Cauchy problem:

Problem 3.1 Find $w \in \mathcal{W}$ such that

$$
\begin{aligned}
& \left.w^{\prime}(t)+A(t, w(t))+\partial \psi(t, w(t)) \ni f(t) \text { a.e. } t \in\right] 0, T[ \\
& w(0)=w_{0} .
\end{aligned}
$$

Here, $\partial \psi$ denotes the Clarke generalized gradient of a locally Lipschitz function $\psi(t, \cdot)$. We recall that a function $w \in \mathcal{W}$ is a solution of Problem 3.1, if there exists $w^{*} \in \mathcal{V}^{*}$ such that $w^{\prime}(t)+A(t, w(t))+w^{*}(t)=f(t)$ a.e. $\left.t \in\right] 0, T\left[, w^{*}(t) \in\right.$ $\partial \psi(t, w(t))$ a.e. $t \in] 0, T\left[\right.$, and $w(0)=w_{0}$.

In the study of Problem 3.1, we need the following hypotheses:

$H(A): \quad A:] 0, T\left[\times V \rightarrow V^{*}\right.$ is such that

(i) $A(\cdot, v)$ is measurable on $] 0, T[$ for all $v \in V$.

(ii) $A(t, \cdot)$ is demicontinuous on $V$ for a.e. $t \in] 0, T[$.

(iii) $\|A(t, v)\|_{V^{*}} \leq a_{0}(t)+a_{1}\|v\|_{V}$ for all $v \in V$, a.e. $\left.t \in\right] 0, T[$ with $a_{0} \in L^{2}(0, T), a_{0} \geq 0$ and $a_{1} \geq 0$.

(iv) $A(t, \cdot)$ is strongly monotone for a.e. $t \in] 0, T\left[\right.$, i.e., for a constant $m_{A}>0$,

$$
\left\langle A\left(t, v_{1}\right)-A\left(t, v_{2}\right), v_{1}-v_{2}\right\rangle_{V^{*} \times V} \geq m_{A}\left\|v_{1}-v_{2}\right\|_{V}^{2}
$$

for all $v_{1}, v_{2} \in V$, a.e. $\left.t \in\right] 0, T[$.

$H(\psi): \quad \psi:] 0, T[\times V \rightarrow \mathbb{R}$ is such that 
(i) $\psi(\cdot, v)$ is measurable on $] 0, T$ [ for all $v \in V$.

(ii) $\psi(t, \cdot)$ is locally Lipschitz on $V$ for a.e. $t \in] 0, T[$.

(iii) $\|\partial \psi(t, v)\|_{V^{*}} \leq c_{0}(t)+c_{1}\|v\|_{V}$ for all $v \in V$, a.e. $\left.t \in\right] 0, T[$

with $c_{0} \in L^{2}(0, T), c_{0} \geq 0, c_{1} \geq 0$.

(iv) $\partial \psi(t, \cdot)$ is relaxed monotone for a.e. $t \in] 0, T$, i.e., for a constant $m_{\psi} \geq 0$,

$$
\left\langle z_{1}-z_{2}, v_{1}-v_{2}\right\rangle_{V^{*} \times V} \geq-m_{\psi}\left\|v_{1}-v_{2}\right\|_{V}^{2}
$$

for all $z_{i} \in \partial \psi\left(t, v_{i}\right), z_{i} \in V^{*}, v_{i} \in V, i=1,2$, a.e. $\left.t \in\right] 0, T[$.

$\left(H_{0}\right): \quad f \in \mathcal{V}^{*}, w_{0} \in V$.

$\overline{\left(H_{1}\right)}: \quad m_{A}>m_{\psi}$.

We have the following existence and uniqueness result.

Theorem 3.1 Under hypotheses $H(A), H(\psi),\left(H_{0}\right)$, and $\left(H_{1}\right)$, Problem 3.1 has the unique solution.

The proof of Theorem 3.1 is similar to [30, Theorem 6], and, therefore, it will be omitted here. Note that the existence and uniqueness result of Theorem 3.1 was proved earlier in [32] under more restrictive smallness assumptions. Theorem 3.1 is of independent interest and can be used in the study of various evolution problems. We apply this theorem to examine the following evolution inclusion of subdifferential type with history-dependent operators.

Problem 3.2 Find $w \in \mathcal{W}$ such that

$$
\begin{aligned}
& w^{\prime}(t)+A(t, w(t))+\left(R_{1} w\right)(t)+N^{*} \partial \varphi(t,(R w)(t), N w(t)) \ni f(t) \\
& \quad \text { a.e. } t \in] 0, T[ \\
& w(0)=w_{0} .
\end{aligned}
$$

Here, $\partial \varphi$ represents the subdifferential of a convex and lower semicontinuous function $\varphi(t, y, \cdot)$, and two nonlinear operators $R$ and $R_{1}$ are assumed to be history dependent, that is, they satisfy condition $\left(\mathrm{H}_{2}\right)$ below.

We introduce the following hypotheses on the data of Problem 3.2. Let $Y$ and $U$ be separable and reflexive Banach spaces.

$\underline{H(\varphi)}: \varphi:] 0, T[\times Y \times U \rightarrow \mathbb{R}$ is such that

(i) $\varphi(\cdot, y, u)$ is measurable on $] 0, T$ [ for all $y \in Y, u \in U$.

(ii) $\varphi(t, \cdot, u)$ is continuous on $Y$ for all $u \in U$, a.e. $t \in] 0, T[$.

(iii) $\varphi(t, y, \cdot)$ is convex and 1.s.c. on $U$ for all $y \in Y$, a.e. $t \in] 0, T[$.

(iv) $\|\partial \varphi(t, y, u)\|_{U^{*}} \leq c_{0 \varphi}(t)+c_{1 \varphi}\|y\|_{Y}+c_{2 \varphi}\|u\|_{U}$ for all $y \in Y, u \in U$, a.e. $t \in] 0, T\left[\right.$ with $c_{0 \varphi} \in L^{2}(0, T), c_{0 \varphi}, c_{1 \varphi}, c_{2 \varphi} \geq 0$.

(v) $\varphi\left(t, y_{1}, u_{2}\right)-\varphi\left(t, y_{1}, u_{1}\right)+\varphi\left(t, y_{2}, u_{1}\right)-\varphi\left(t, y_{2}, u_{2}\right)$

$\leq \beta_{\varphi}\left\|y_{1}-y_{2}\right\|_{Y}\left\|u_{1}-u_{2}\right\|_{U}$ for all $y_{i} \in Y, u_{i} \in U, i=1$, 2, a.e. $\left.t \in\right] 0, T[$ with $\beta_{\varphi} \geq 0$.

$H(N): \quad N \in \mathcal{L}(V, U)$.

$\overline{\left(H_{2}\right)}: \quad R: \mathcal{V} \rightarrow L^{2}(0, T ; Y)$ and $R_{1}: \mathcal{V} \rightarrow \mathcal{V}^{*}$ are such that 
(i) $\left\|\left(R v_{1}\right)(t)-\left(R v_{2}\right)(t)\right\|_{Y} \leq c_{R} \int_{0}^{t}\left\|v_{1}(s)-v_{2}(s)\right\|_{V} \mathrm{~d} s$ for all $v_{1}, v_{2} \in \mathcal{V}$, a.e. $t \in] 0, T\left[\right.$ with $c_{R}>0$.

(ii) $\left\|\left(R_{1} v_{1}\right)(t)-\left(R_{1} v_{2}\right)(t)\right\|_{V^{*}} \leq c_{R_{1}} \int_{0}^{t}\left\|v_{1}(s)-v_{2}(s)\right\|_{V} \mathrm{~d} s$ for all $v_{1}, v_{2} \in \mathcal{V}$, a.e. $t \in] 0, T\left[\right.$ with $c_{R_{1}}>0$.

Exploiting Theorem 3.1 and using ideas from [32, Theorem 5] and [30, Theorem 9], we obtain the following result.

Theorem 3.2 Under hypotheses $H(A), H(\varphi), H(N),\left(H_{0}\right)$, and $\left(H_{2}\right)$, Problem 3.2 has a unique solution.

\section{Continuous Dependence Result}

In this section, we provide the continuous dependence result for the evolution inclusion in Problem 3.2. We are interested in the continuity, in the weak topologies, of the map, which to the right-hand side and initial condition in Problem 3.2 assigns its unique solution.

We first establish some a priori bounds for the solution.

Proposition 4.1 Under hypotheses of Theorem 3.2, if $w \in \mathcal{W}$ is a solution to Problem 3.2, then there exists a constant $c>0$ such that

$\|w\|_{C(0, T ; H)}+\|w\|_{\mathcal{W}} \leq c\left(1+\left\|w_{0}\right\|_{V}+\|f\|_{\mathcal{V}^{*}}+\left\|R 0_{\mathcal{V}}\right\|_{L^{2}(0, T ; Y)}+\left\|R_{1} 0 \mathcal{V}\right\|_{L^{2}\left(0, T ; V^{*}\right)}\right)$

where $0 \mathcal{V}$ denotes the zero element of $\mathcal{V}$.

Proof We denote by $w \in \mathcal{W}$ a solution to Problem 3.2. This means that there exists $\eta \in L^{2}\left(0, T ; U^{*}\right)$ such that

$$
\begin{aligned}
& \left.w^{\prime}(t)+A(t, w(t))+\left(R_{1} w\right)(t)+N^{*} \eta(t)=f(t) \text { a.e. } t \in\right] 0, T[ \\
& \eta(t) \in \partial \varphi(t,(R w)(t), N w(t)) \text { a.e. } t \in] 0, T[ \\
& w(0)=w_{0} .
\end{aligned}
$$

Taking the duality in (2) with $w(t)$, we have

$$
\begin{aligned}
& \left\langle w^{\prime}(t)+A(t, w(t))+\left(R_{1} w\right)(t), w(t)\right\rangle_{V^{*} \times V}+\langle\eta(t), N w(t)\rangle_{Y^{*} \times Y} \\
& \quad=\langle f(t), w(t)\rangle_{V^{*} \times V}
\end{aligned}
$$

for a.e. $t \in] 0, T$ [. In the bounds below, we repeatedly use Hölder's inequality, Young's inequality $a b \leq \frac{\varepsilon^{2}}{2} a^{2}+\frac{1}{2 \varepsilon^{2}} b^{2}$ with $\varepsilon>0$, and the elementary inequality $(a+b)^{2} \leq$ $2\left(a^{2}+b^{2}\right)$ for all $a, b \in \mathbb{R}$. Let $t \in[0, T]$ and $\varepsilon>0$. From hypothesis $\left(H_{2}\right)$, we have 


$$
\begin{aligned}
& \|(R w)(t)\|_{Y}^{2} \leq 2 c_{R}^{2} t \int_{0}^{t}\|w(s)\|_{V}^{2} \mathrm{~d} s+2\left\|\left(R 0_{\mathcal{V}}\right)(t)\right\|_{Y}^{2}, \\
& \left\|\left(R_{1} w\right)(t)\right\|_{V^{*}}^{2} \leq 2 c_{R_{1}}^{2} t \int_{0}^{t}\|w(s)\|_{V}^{2} \mathrm{~d} s+2\left\|\left(R_{1} 0_{\mathcal{V}}\right)(t)\right\|_{V^{*}}^{2}
\end{aligned}
$$

By the assumption $H(\varphi)$ (iv) and (6), we obtain

$$
\begin{aligned}
& \left|\langle\partial \varphi(t,(R w)(t), 0), N w(t)\rangle_{Y^{*} \times Y}\right| \leq\left(c_{0 \varphi}(t)+c_{1 \varphi}\|(R w)(t)\|_{Y}\right)\|N\|\|w(t)\|_{V} \\
& \quad \leq \frac{\varepsilon^{2}}{2}\|N\|^{2}\|w(t)\|_{V}^{2}+\frac{1}{\varepsilon^{2}}\left(c_{0 \varphi}^{2}(t)+c_{1 \varphi}^{2}\|(R w)(t)\|_{Y}^{2}\right) \\
& \quad \leq \frac{\varepsilon^{2}}{2}\|N\|^{2}\|w(t)\|_{V}^{2}+\frac{c_{0 \varphi}^{2}(t)}{\varepsilon^{2}}+\frac{2 c_{1 \varphi}^{2} c_{R}^{2} T}{\varepsilon^{2}} \int_{0}^{t}\|w(s)\|_{V}^{2} \mathrm{~d} s+\frac{2 c_{1 \varphi}^{2}}{\varepsilon^{2}}\|(R 0 \mathcal{V})(t)\|_{Y}^{2} .
\end{aligned}
$$

The latter, combined with (3) and the monotonicity of the convex subdifferential, yields

$$
\begin{aligned}
& \langle\eta(t), N w(t)\rangle_{Y^{*} \times Y}=\langle\partial \varphi(t,(R w)(t), N w(t)), N w(t)\rangle_{Y^{*} \times Y} \\
& \geq\langle\partial \varphi(t,(R w)(t), 0), N w(t)\rangle_{Y^{*} \times Y} \geq-\frac{\varepsilon^{2}}{2}\|N\|^{2}\|w(t)\|_{V}^{2} \\
& \quad-\frac{c_{0 \varphi}^{2}(t)}{\varepsilon^{2}}-\frac{2 c_{1 \varphi}^{2} c_{R}^{2} T}{\varepsilon^{2}} \int_{0}^{t}\|w(s)\|_{V}^{2} \mathrm{~d} s-\frac{2 c_{1 \varphi}^{2}}{\varepsilon^{2}}\left\|\left(R 0_{\mathcal{V}}\right)(t)\right\|_{Y}^{2} .
\end{aligned}
$$

From inequality (7), we have

$$
\begin{aligned}
& \left.\int_{0}^{t}\left\langle\left(R_{1} w\right)(s)\right), w(s)\right\rangle_{V^{*} \times V} \mathrm{~d} s \leq \frac{\varepsilon^{2}}{2} \int_{0}^{t}\|w(s)\|_{V}^{2} \mathrm{~d} s \\
& +\frac{c_{R_{1}}^{2} t}{\varepsilon^{2}} \int_{0}^{t}\left(\int_{0}^{s}\|w(\tau)\|_{V}^{2} \mathrm{~d} \tau\right) \mathrm{d} s+\frac{1}{\varepsilon^{2}} \int_{0}^{t}\left\|\left(R_{1} 0 \mathcal{V}\right)(s)\right\|_{V^{*}}^{2} \mathrm{~d} s .
\end{aligned}
$$

On the other hand, a simple calculation gives

$$
\begin{aligned}
& \left.\int_{0}^{t}\langle f(s)), w(s)\right\rangle_{V^{*} \times V} \mathrm{~d} s \leq \frac{\varepsilon^{2}}{2} \int_{0}^{t}\|w(s)\|_{V}^{2} \mathrm{~d} s+\frac{1}{2 \varepsilon^{2}} \int_{0}^{t}\|f(s)\|_{V^{*}}^{2} \mathrm{~d} s, \\
& \int_{0}^{t} a_{0}(s)\|w(s)\|_{V} \mathrm{~d} s \leq \frac{\varepsilon^{2}}{2}\|w\|_{L^{2}(0, t ; V)}^{2}+\frac{1}{2 \varepsilon^{2}}\left\|a_{0}\right\|_{L^{2}(0, T)}^{2} .
\end{aligned}
$$

Next, we integrate (5) on ]0, $t$ [ for all $t \in[0, T]$, use the integration by parts formula in [36, Proposition 8.4.14], $H(A)$ (iii) and (iv), and the inequalities (8)-(11), to deduce

$$
\begin{gathered}
\frac{1}{2}\|w(t)\|_{H}^{2}+\left(m_{A}-\frac{3 \varepsilon^{2}}{2}-\frac{\varepsilon^{2}}{2}\|N\|^{2}\right) \int_{0}^{t}\|w(s)\|_{V}^{2} \mathrm{~d} s \\
\quad \leq \frac{1}{2}\left\|w_{0}\right\|_{H}^{2}+\frac{1}{2 \varepsilon^{2}}\left\|a_{0}\right\|_{L^{2}(0, T)}^{2}+\frac{1}{\varepsilon^{2}}\left\|c_{0 \varphi}\right\|_{L^{2}(0, T)}^{2}
\end{gathered}
$$




$$
\begin{aligned}
& +\frac{1}{\varepsilon^{2}}\left(2 c_{1 \varphi}^{2} c_{R}^{2} T+c_{R_{1}}^{2} T\right) \int_{0}^{t}\left(\int_{0}^{s}\|w(\tau)\|_{V}^{2} \mathrm{~d} \tau\right) \mathrm{d} s+\frac{1}{2 \varepsilon^{2}} \int_{0}^{t}\|f(s)\|_{V^{*}}^{2} \mathrm{~d} s \\
& +\frac{2 c_{1 \varphi}^{2}}{\varepsilon^{2}} \int_{0}^{t}\|(R 0 \mathcal{V})(s)\|_{Y}^{2} \mathrm{~d} s+\frac{1}{\varepsilon^{2}} \int_{0}^{t}\left\|\left(R_{1} 0 \mathcal{V}\right)(s)\right\|_{V^{*}}^{2} \mathrm{~d} s .
\end{aligned}
$$

Now, we choose $\varepsilon>0$ such that

$$
m_{A}-\frac{3 \varepsilon^{2}}{2}-\frac{\varepsilon^{2}}{2}\|N\|^{2}>0 .
$$

Hence, for some positive constants $d_{i}, i=1, \ldots, 5$, we have

$$
\begin{gathered}
d_{1} \int_{0}^{t}\|w(s)\|_{V}^{2} \mathrm{~d} s \leq \\
\frac{1}{2}\left\|w_{0}\right\|_{H}^{2}+d_{2}\|f\|_{L^{2}\left(0, t ; V^{*}\right)}^{2}+d_{3} \int_{0}^{t}\left(\int_{0}^{s}\|w(\tau)\|_{V}^{2} \mathrm{~d} \tau\right) \mathrm{d} s \\
+d_{4} \int_{0}^{t}\left(\left\|\left(R 0_{\mathcal{V}}\right)(s)\right\|_{Y}^{2}+\left\|\left(R_{1} 0_{\mathcal{V}}\right)(s)\right\|_{V^{*}}^{2}\right) \mathrm{d} s+d_{5}
\end{gathered}
$$

for all $t \in[0, T]$. Invoking Gronwall's inequality, see, e.g., [31, Lemma 2.7], we deduce the desired estimate on the term $\|w\| \mathcal{V}$. Directly from Eq. (2), we obtain the bound on $\left\|w^{\prime}\right\| \mathcal{V}^{*}$ and finally also on the norm of the solution in $C(0, T ; H)$. This proves the bound in the statement of the proposition and completes the proof.

To discuss the continuous dependence, we need stronger versions of the hypotheses introduced in the previous section. In particular, the operator $A$ is assumed to be time independent and weakly-weakly continuous, which obviously implies the demicontinuity in $H(A)$ (ii).

$H(A)_{1}: \quad A: V \rightarrow V^{*}$ is such that

(i) $A$ is weakly-weakly continuous.

(ii) $\|A v\|_{V^{*}} \leq a_{0}+a_{1}\|v\|_{V}$ for all $v \in V$ with $a_{0}, a_{1} \geq 0$.

(iii) $A$ is strongly monotone with constant $m_{A}>0$, i.e.,

$$
\left\langle A v_{1}-A v_{2}, v_{1}-v_{2}\right\rangle_{V^{*} \times V} \geq m_{A}\left\|v_{1}-v_{2}\right\|_{V}^{2} \text { for all } v_{1}, v_{2} \in V \text {. }
$$

$\underline{H(N)_{1}}: \quad N \in \mathcal{L}(V, U)$ and its Nemytskii operator $\mathcal{N}: \mathcal{W} \subset \mathcal{V} \rightarrow L^{2}(0, T ; U)$ is compact.

$\underline{\left(H_{4}\right)}: \quad R$ and $R_{1}$ satisfy $\left(H_{2}\right)$, and

(i) $R: \mathcal{W} \subset \mathcal{V} \rightarrow L^{2}(0, T ; Y)$ is compact.

(ii) $R_{1}: \mathcal{V} \rightarrow \mathcal{V}^{*}$ is weakly-weakly continuous.

(iii) $\left(R 0 \mathcal{V}, R_{1} 0_{\mathcal{V}}\right)$ remains in a bounded subset of $L^{2}\left(0, T ; Y \times V^{*}\right)$.

Theorem 4.1 If hypotheses $H(A)_{1}, H(\varphi), H(N)_{1},\left(H_{0}\right)$, and $\left(H_{4}\right)$ hold, $\left\{f_{n}\right\} \subset \mathcal{V}^{*}$, $f_{n} \rightarrow f$ weakly in $\mathcal{V}^{*},\left\{w_{0}^{n}\right\} \subset V, w_{0}^{n} \rightarrow w_{0}$ weakly in $V$, and $\left\{w_{n}\right\} \subset \mathcal{W}, w \in \mathcal{W}$ are the unique solutions to Problem 3.2 corresponding to $\left\{\left(f_{n}, w_{0}^{n}\right)\right\}$ and $\left(f, w_{0}\right)$, respectively, then $w_{n} \rightarrow w$ weakly in $\mathcal{W}$, as $n \rightarrow \infty$. 
Proof The unique solvability of Problem 3.2 follows from Theorem 3.2. We prove the continuous dependence result. Let $\left\{f_{n}\right\} \subset \mathcal{V}^{*}, f_{n} \rightarrow f$ weakly in $\mathcal{V}^{*},\left\{w_{0}^{n}\right\} \subset V$, $w_{0}^{n} \rightarrow w_{0}$ weakly in $V$, and $\left\{w_{n}\right\} \subset \mathcal{W}$ be the unique solution to Problem 3.2 corresponding to $\left\{\left(f_{n}, w_{0}^{n}\right)\right\}, n \in \mathbb{N}$. Then, there is a sequence $\left\{\eta_{n}\right\} \subset L^{2}\left(0, T ; U^{*}\right)$ such that

$$
\begin{aligned}
& \left.w_{n}^{\prime}(t)+A w_{n}(t)+\left(R_{1} w_{n}\right)(t)+N^{*} \eta_{n}(t)=f_{n}(t) \text { a.e. } t \in\right] 0, T[, \\
& \left.\eta_{n}(t) \in \partial \varphi\left(t,\left(R w_{n}\right)(t), N w_{n}(t)\right) \text { a.e. } t \in\right] 0, T[ \\
& w_{n}(0)=w_{0}^{n} .
\end{aligned}
$$

Thus, by Proposition 4.1 combined with $\left(H_{4}\right)$ (iii), we know that the sequence $\left\{w_{n}\right\}$ is uniformly bounded in $\mathcal{W}$. From the reflexivity of $\mathcal{W}$, by passing to a subsequence if necessary, we may assume that $w_{n} \rightarrow w$ weakly in $\mathcal{W}$ with $w \in \mathcal{W}$, as $n \rightarrow \infty$. It remains to prove that $w$ is the unique solution in $\mathcal{W}$ to Problem 3.2 corresponding to $\left(f, w_{0}\right)$.

Using an argument similar to [39, Lemma 13], from the hypothesis $H(A)_{1}$, we known that

$$
\mathcal{A} w_{n} \rightarrow \mathcal{A} w \text { weakly in } \mathcal{V}^{*} \text {, as } n \rightarrow \infty .
$$

By the assumption $\left(H_{4}\right)(\mathrm{ii})$, it follows

$$
R_{1} w_{n} \rightarrow R_{1} w \text { weakly in } \mathcal{V}^{*} \text {, as } n \rightarrow \infty
$$

Further, we use $H(N)_{1}$ to get $\mathcal{N} w_{n} \rightarrow \mathcal{N} w$ in $L^{2}(0, T ; U)$, which, for a next subsequence if necessary, entails

$$
\left.N w_{n}(t) \rightarrow N w(t) \text { in } U \text {, for a.e. } t \in\right] 0, T[\text {. }
$$

On the other hand, by $\left(H_{4}\right)(\mathrm{i})$, we have $R w_{n} \rightarrow R w$ in $L^{2}(0, T ; Y)$. Hence, again at least for a subsequence, we may suppose that

$$
\left.\left(R w_{n}\right)(t) \rightarrow(R w)(t) \text { in } Y, \text { for a.e. } t \in\right] 0, T[
$$

Claim If $\varphi:(0, T) \times Y \times U \rightarrow \mathbb{R}$ satisfies $H(\varphi)(i i)-(i v)$, then the multivalued map

$$
Y \times U \ni(y, u) \mapsto \partial \varphi(t, y, u) \subset U^{*}
$$

is upper semicontinuous from $Y \times U$ into subsets of $U^{*}$ endowed with the weak topology, with nonempty, closed, and convex values, for a.e. $t \in] 0, T[$.

Indeed, since $\varphi$ has finite values, we apply [36, Proposition 6.3.10] to see that the map $\partial \varphi(t, \cdot, \cdot)$, for a.e. $t \in] 0, T$, has nonempty, closed, and convex values. Let $t \in] 0, T\left[\backslash Z_{1}\right.$ with $m\left(Z_{1}\right)=0, E \subset U^{*}$ be weakly closed, and let

$$
(\partial \varphi)^{-}(E):=\{(y, u) \in Y \times U: \partial \varphi(t, y, u) \cap E \neq \emptyset\} .
$$


Let $\left\{\left(y_{n}, u_{n}\right)\right\} \subset(\partial \varphi)^{-}(E)$ be such that $\left(y_{n}, u_{n}\right) \rightarrow(y, u)$ in $Y \times U$, as $n \rightarrow \infty$. We can find $\left\{\rho_{n}\right\} \subset U^{*}$ such that $\rho_{n} \in \partial \varphi\left(t, y_{n}, u_{n}\right) \cap E$ for each $n \in \mathbb{N}$. It follows from $H(\varphi)$ (iv) that the sequence $\left\{\rho_{n}\right\}$ is bounded in $U^{*}$, which by the reflexivity of $U^{*}$ implies, at least for a subsequence, that $\rho_{n} \rightarrow \rho$ weakly in $U^{*}$. Clearly, we have $\rho \in E$, and

$$
\left\langle\rho_{n}, w-u_{n}\right\rangle_{U^{*} \times U} \leq \varphi\left(t, y_{n}, w\right)-\varphi\left(t, y_{n}, u_{n}\right) \text { for all } w \in U
$$

From [34, Proposition 5.2.10], we know that $\varphi(t, y, \cdot)$ is locally Lipschitz, for all $y \in Y$. Then exploiting $H(\varphi)$ (ii)-(iv) and [31, Lemma 3.43], we obtain that $\varphi(t, \cdot, \cdot)$ is continuous on $Y \times U$. This allows one to pass to the limit

$$
\begin{aligned}
& \langle\rho, w-u\rangle_{U^{*} \times U}=\limsup _{n \rightarrow \infty}\left\langle\rho_{n}, w-u_{n}\right\rangle_{U^{*} \times U} \\
& \quad \leq \limsup _{n \rightarrow \infty}\left(\varphi\left(t, y_{n}, w\right)-\varphi\left(y, y_{n}, u_{n}\right)\right)=\varphi(t, y, w)-\varphi(t, y, u)
\end{aligned}
$$

for all $w \in U$. Hence $\rho \in \partial \varphi(t, y, u) \cap E$ and also $(y, u) \in(\partial \varphi)^{-}(E)$. This means that the set $(\partial \varphi)^{-}(E)$ is closed in $Y \times U$, which by [34, Proposition 4.1.4] implies the desired upper semicontinuity of $\partial \varphi(t, \cdot, \cdot)$, for a.e. $t \in] 0, T[$, and proves the claim.

Next, we treat the inclusion (13). The main tool is the convergence theorem of Aubin-Cellina [40, p.60], in a version provided in [41, Proposition 2] or [42, Lemma 2.6]. By $H(\varphi)$ (iv), it follows that the sequence $\left\{\eta_{n}\right\}$ is uniformly bounded in $L^{2}\left(0, T ; U^{*}\right)$. Hence, again, by passing to a subsequence if necessary, we may assume that

$$
\eta_{n} \rightarrow \eta \text { weakly in } L^{2}\left(0, T ; U^{*}\right)
$$

with $\eta \in L^{2}\left(0, T ; U^{*}\right)$. Using the convergences (17)-(19) and the claim, we apply the aforementioned convergence theorem to deduce

$$
\eta(t) \in \partial \varphi(t,(R w)(t), N w(t)) \text { a.e. } t \in] 0, T[.
$$

On the other hand, it is immediate to see that

$$
\mathcal{N}^{*} \eta_{n} \rightarrow \mathcal{N} \eta \text { weakly in } \mathcal{V}^{*}
$$

Further, observe that the map

$$
\mathcal{W} \ni w \mapsto w(0) \in H
$$

is linear and continuous, and hence, the convergence $w_{n} \rightarrow w$ weakly in $\mathcal{W}$ entails $w_{n}(0) \rightarrow w(0)$ weakly in $H$. Passing in (14) to the weak limit in $H$, we immediately get $w(0)=w_{0}$. Finally, invoking convergences $w_{n}^{\prime} \rightarrow w^{\prime}$ weakly in $\mathcal{V}^{*},(15),(16)$, (21), and $f_{n} \rightarrow f$ weakly in $\mathcal{V}^{*}$, and passing to the limit in the equation $w_{n}^{\prime}+\mathcal{A} w_{n}+$ $R_{1} w_{n}+\mathcal{N}^{*} \eta_{n}=f_{n}$ in $\mathcal{V}^{*}$, as $n \rightarrow \infty$, we have

$$
w^{\prime}+\mathcal{A} w+R_{1} w+\mathcal{N}^{*} \eta=f \text { in } \mathcal{V}^{*} .
$$


The latter, combined with (20) and $w(0)=w_{0}$, implies that $w \in \mathcal{W}$ is the solution to Problem 3.2 corresponding to $\left(f, w_{0}\right)$. Since the solution is unique, we conclude that the whole sequence $\left\{w_{n}\right\}$ converges to $w$ weakly in $\mathcal{W}$. This completes the proof of the theorem.

\section{Optimal Control Problem}

In this section, we provide an application of the continuous dependence result to study an optimal control problem for the evolution inclusion with history-dependent operators.

Given a separable, reflexive Banach space $X$ of controls, an element $\tilde{f} \in \mathcal{V}^{*}$, and a family of operators $B:] 0, T\left[\rightarrow \mathcal{L}\left(X, V^{*}\right)\right.$, we consider the following controlled evolution inclusion.

Problem 5.1 Find $\left(w, v, w_{0}\right) \in \mathcal{W} \times L^{2}(0, T ; X) \times V$ such that

$$
\begin{aligned}
& w^{\prime}(t)+A w(t)+\left(R_{1} w\right)(t)+N^{*} \partial \varphi(t,(R w)(t), N w(t)) \ni \widetilde{f}(t)+B(t) v(t) \\
& \quad \text { a.e. } t \in] 0, T[, \\
& \left.w(0)=w_{0}, v(t) \in U_{a d}(t) \text { a.e. } t \in\right] 0, T\left[, v(\cdot) \text { measurable, } w_{0} \in V_{a d} .\right.
\end{aligned}
$$

In this problem, $\left(v, w_{0}\right) \in L^{2}(0, T ; X) \times V$ denotes the control pair, and $w \in \mathcal{W}$ is the corresponding state (solution) of the system. The sets $U_{a d}(\cdot)$ and $V_{a d}$ represent the constraints sets of admissible controls.

Consider the following Bolza-type optimal control problem:

$$
J\left(w, v, w_{0}\right)=l_{1}\left(w_{0}\right)+l_{2}(w(T))+\int_{0}^{T} L(t, w(t), v(t)) \mathrm{d} t \longrightarrow \text { inf }=: m
$$

in the class of triples $\left(w, v, w_{0}\right) \in \mathcal{W} \times L^{2}(0, T ; X) \times V$ satisfying Problem 5.1.

We will need the hypotheses on the data of problem (22).

$H(B): \quad B \in L^{\infty}\left(0, T ; \mathcal{L}\left(X, V^{*}\right)\right), \widetilde{f} \in \mathcal{V}^{*}$.

$\overline{H\left(V_{a d}\right)}: V_{a d}$ is a bounded, closed, convex subset of $V$.

$\left.\overline{H\left(U_{a d}\right)}: \quad U_{a d}:\right] 0, T\left[\rightrightarrows 2^{X} \backslash\{\emptyset\}\right.$ is a map with closed, convex values such that

$$
t \mapsto\left\|U_{a d}(t)\right\|=\sup \left\{\|v\|_{X}: v \in U_{a d}(t)\right\} \text { belongs to } L^{2}(0, T)
$$

$\underline{H\left(l_{1}, l_{2}\right)}: \quad l_{1}: V \rightarrow \mathbb{R}$ and $l_{2}: H \rightarrow \mathbb{R}$ are sequentially weakly lower semicontinuous.

$\underline{H(L)}: \quad L:] 0, T[\times H \times X \rightarrow \mathbb{R} \cup\{\infty\}$ is $\mathcal{T} \times \mathcal{B}(H \times X)$-measurable function such that

(i) $L(t, w, \cdot)$ is convex on $X$, for all $w \in H$, a.e. $t \in] 0, T[$.

(ii) $L(t, \cdot, \cdot)$ is sequentially lower semicontinuous on $H \times X$, a.e. $t \in] 0, T[$. 
(iii) There is $M>0$ and $\psi \in L^{1}(0, T)$ such that

$$
\left.L(t, w, v) \geq \psi(t)-M\left(\|w\|_{H}+\|v\|_{X}\right) \text { for all } w \in H, v \in X \text {, a.e. } t \in\right] 0, T[\text {. }
$$

We introduce the set of admissible state-control triples defined by

$\mathbb{S}=\left\{\left(w, v, w_{0}\right) \in \mathcal{W} \times L^{2}(0, T ; X) \times V:\left(w, v, w_{0}\right)\right.$ satisfies Problem 5.1$\}$

As a consequence of Theorem 3.2, we obtain the following property.

Lemma 5.1 If hypotheses $H(A)_{1}, H(\varphi), H(N)_{1},\left(H_{0}\right),\left(H_{4}\right)$, and $H(B)$ hold, then $\mathbb{S} \neq \emptyset$.

Next, we introduce the set of optimal state-control triples defined by

$$
\mathbb{O}=\left\{\left(w, v, w_{0}\right) \in \mathcal{W} \times L^{2}(0, T ; X) \times V: J\left(w, v, w_{0}\right)=m\right\} .
$$

Since the integrand $L$ of the cost is extended valued, we need the following feasibility hypothesis:

$H$ (feas) : there exists $\left(w, v, w_{0}\right) \in \mathbb{S}$ such that $J\left(w, v, w_{0}\right)<\infty$.

Using [34, Proposition 4.7.44], [43, Theorem 2.1], and Theorem 4.1, and applying the direct method of the calculus of variations, we deduce:

Theorem 5.1 Assume hypotheses of Lemma 5.1, $H\left(V_{a d}\right), H\left(U_{a d}\right), H\left(l_{1}, l_{2}\right), H(L)$, and $H$ (feas). Then, $\mathbb{O}$ is a nonempty subset of $\mathcal{W} \times L^{2}(0, T ; X) \times V$.

\section{Application to a Frictional Contact Problem}

In this section, we illustrate the applicability of our results of Sects. 3-5 and work out, in detail, a nonlinear optimal control problem for a dynamic viscoelastic contact problem with friction. The latter can be formulated, for instance, as the historydependent evolution inclusion or the history-dependent variational inequality. Note that the contact problem considered below has been examined earlier in [32], where the existence and uniqueness result was obtained under more restrictive hypotheses.

We recall shortly the necessary notation and the weak formulation of the problem, already obtained in [32]. We skip the model description and its physical interpretation since they can be found in [32]; see also [18,19,31].

Let $\Omega$ be a regular domain in $\mathbb{R}^{d}, d=2,3$, occupied in its reference configuration by a viscoelastic body with long memory. The boundary $\partial \Omega=\Gamma$ consists of three disjoint measurable parts $\Gamma_{D}, \Gamma_{N}$, and $\Gamma_{N}$ such that the measure $m\left(\Gamma_{D}\right)>0$. The body is clamped on $\Gamma_{D}$ (the displacement field vanishes there), the surface tractions act on $\Gamma_{N}$, while $\Gamma_{C}$ is a contact surface. The volume forces of density $\boldsymbol{f}_{0}$ act in the body $\Omega$, and surface tractions of density $\boldsymbol{f}_{N}$ act on part $\Gamma_{N}$. These forces depend on time. We use $\boldsymbol{u}=\left(u_{i}\right), \boldsymbol{\sigma}=\left(\sigma_{i j}\right)$, and $\boldsymbol{\varepsilon}(\boldsymbol{u})=\left(\varepsilon_{i j}(\boldsymbol{u})\right)$ to denote the displacement 
vector, the stress tensor, and the linearized strain tensor, respectively. The latter is defined by

$$
\varepsilon_{i j}(\boldsymbol{u})=\frac{1}{2}\left(u_{i, j}+u_{j, i}\right),
$$

where $u_{i, j}=\partial u_{i} / \partial x_{j}$. For a vector field, the notation $v_{v}$ and $\boldsymbol{v}_{\tau}$ stands for the normal and tangential components of $\boldsymbol{v}$ on $\partial \Omega$, given by $v_{\nu}=\boldsymbol{v} \cdot \boldsymbol{v}$ and $\boldsymbol{v}_{\tau}=\boldsymbol{v}-\boldsymbol{v}_{\nu} \boldsymbol{v}$, where $v$ is the outward unit normal on the boundary. The normal and tangential components of the stress field $\boldsymbol{\sigma}$ on the boundary are defined by $\sigma_{v}=(\boldsymbol{\sigma} \boldsymbol{v}) \cdot \boldsymbol{v}$ and $\sigma_{\tau}=\boldsymbol{\sigma} \boldsymbol{v}-\sigma_{\nu} \boldsymbol{v}$, respectively. The symbol $\mathbb{S}^{d}$ denotes the space of symmetric matrices of order $d$, and the canonical inner products on $\mathbb{R}^{d}$ and $\mathbb{S}^{d}$ are given by $\boldsymbol{u} \cdot \boldsymbol{v}=u_{i} v_{i}$ for all $\boldsymbol{u}=\left(u_{i}\right)$, $\boldsymbol{v}=\left(v_{i}\right) \in \mathbb{R}^{d}$, and $\boldsymbol{\sigma} \cdot \boldsymbol{\tau}=\sigma_{i j} \tau_{i j}$ for all $\boldsymbol{\sigma}=\left(\sigma_{i j}\right), \boldsymbol{\tau}=\left(\tau_{i j}\right) \in \mathbb{S}^{d}$, respectively. All indices $i, j, k, l$ run between 1 and $d$, and, unless stated otherwise, the summation convention over repeated indices is applied.

We are interested in the evolution process of the mechanical state of the body, in the finite time interval ]0, $T$ [ with $0<T<\infty$. We use the following notation $Q=\Omega \times] 0, T[, \Sigma=\Gamma \times] 0, T\left[, \Sigma_{D}=\Gamma_{D} \times\right] 0, T\left[, \Sigma_{N}=\Gamma_{N} \times\right] 0, T\left[\right.$ and $\Sigma_{C}=$ $\left.\Gamma_{C} \times\right] 0, T$. The classical formulation of dynamic contact problem with friction reads as follows:

Problem $\mathcal{P}$. Find a displacement field $\boldsymbol{u}: Q \rightarrow \mathbb{R}^{d}$ and a stress field $\boldsymbol{\sigma}: Q \rightarrow \mathbb{S}^{d}$ such that

$$
\begin{aligned}
& \boldsymbol{u}^{\prime \prime}(t)-\operatorname{Div} \boldsymbol{\sigma}(t)=\boldsymbol{f}_{0}(t) \quad \text { in } Q, \\
& \boldsymbol{\sigma}(t)=\mathscr{A}\left(t, \boldsymbol{\varepsilon}\left(\boldsymbol{u}^{\prime}(t)\right)\right)+\mathscr{B}(t, \boldsymbol{\varepsilon}(\boldsymbol{u}(t)))+\int_{0}^{t} \mathscr{K}\left(t-s, \boldsymbol{\varepsilon}\left(\boldsymbol{u}^{\prime}(s)\right)\right) \mathrm{d} s \quad \text { in } Q, \\
& \boldsymbol{u}(t)=\mathbf{0} \text { on } \Sigma_{D}, \\
& \boldsymbol{\sigma}(t) \boldsymbol{v}=\boldsymbol{f}_{N}(t) \text { on } \Sigma_{N}, \\
& -\sigma_{\nu}(t)=k\left(\int_{0}^{t} u_{v}(s) \mathrm{d} s\right) p\left(t, u_{v}(t)\right)+\int_{0}^{t} b\left(t-s, u_{v}(s)\right) \mathrm{d} s \text { on } \Sigma_{C}, \\
& \left\|\sigma_{\tau}(t)\right\| \leq \mu\left|\sigma_{\nu}(t)\right|, \quad-\sigma_{\tau}(t)=\mu\left|\sigma_{\nu}(t)\right| \frac{\boldsymbol{u}_{\tau}^{\prime}(t)}{\left\|\boldsymbol{u}_{\tau}^{\prime}(t)\right\|_{\mathbb{R}^{d}}}, \text { if } \boldsymbol{u}_{\tau}^{\prime} \neq 0 \quad \text { on } \Sigma_{C}, \\
& \boldsymbol{u}(0)=\boldsymbol{u}_{0}, \quad \boldsymbol{u}^{\prime}(0)=\boldsymbol{w}_{0} \quad \text { in } \Omega .
\end{aligned}
$$

To state the variational formulation of Problem $\mathcal{P}$, we introduce the evolution triple of spaces $\left(V, H, V^{*}\right)$ with

$$
V=\left\{\boldsymbol{v} \in H^{1}\left(\Omega ; \mathbb{R}^{d}\right): \boldsymbol{v}=0 \text { on } \Gamma_{D}\right\}, \quad H=L^{2}\left(\Omega ; \mathbb{R}^{d}\right) .
$$

The inner product and the norm on $V$ are given by $\langle\boldsymbol{u}, \boldsymbol{v}\rangle_{V}=\langle\boldsymbol{\varepsilon}(\boldsymbol{u}), \boldsymbol{\varepsilon}(\boldsymbol{v})\rangle_{\mathcal{H}},\|\boldsymbol{v}\|=$ $\|\boldsymbol{\varepsilon}(\boldsymbol{v})\|_{\mathcal{H}}$ for $\boldsymbol{u}, \boldsymbol{v} \in V$, where $\mathcal{H}=L^{2}\left(\Omega ; \mathbb{S}^{d}\right)$. Further, by the continuity of the trace operator, we deduce that $V \subset H^{1}\left(\Omega ; \mathbb{R}^{d}\right)$ is a closed subspace. Based on the Korn inequality

$$
\|\boldsymbol{v}\|_{H^{1}\left(\Omega ; \mathbb{R}^{d}\right)} \leq c\|\boldsymbol{\varepsilon}(\boldsymbol{v})\|_{\mathcal{H}} \text { for } \boldsymbol{v} \in V \text { with } c>0
$$


we conclude that the norms $\|\cdot\|_{H^{1}\left(\Omega ; \mathbb{R}^{d}\right)}$ and $\|\cdot\|$ are equivalent on $V$. Recall that the trace operator $\gamma: V \rightarrow L^{2}\left(\Gamma ; \mathbb{R}^{d}\right)$ is linear and continuous. We use the notation $\boldsymbol{v}$ for the trace of $\boldsymbol{v}$ on the boundary $\Gamma$.

We state the following assumptions on operators and functions which occur in Problem $\mathcal{P}$ :

The viscosity operator $\mathscr{A}: Q \times \mathbb{S}^{d} \rightarrow \mathbb{S}^{d}$ is such that

(a) $\mathscr{A}(\cdot, \cdot, \boldsymbol{\varepsilon})$ is measurable on $Q$ for all $\boldsymbol{\varepsilon} \in \mathbb{S}^{d}$.

(b) $\mathscr{A}(\boldsymbol{x}, t, \cdot)$ is continuous on $\mathbb{S}^{d}$ for a.e. $(\boldsymbol{x}, t) \in Q$.

(c) $\left(\mathscr{A}\left(\boldsymbol{x}, t, \boldsymbol{\varepsilon}_{1}\right)-\mathscr{A}\left(\boldsymbol{x}, t, \boldsymbol{\varepsilon}_{2}\right)\right) \cdot\left(\boldsymbol{\varepsilon}_{1}-\boldsymbol{\varepsilon}_{2}\right) \geq m_{\mathscr{A}}\left\|\boldsymbol{\varepsilon}_{1}-\boldsymbol{\varepsilon}_{2}\right\|_{\mathbb{S}^{d}}^{2}$

for all $\boldsymbol{\varepsilon}_{1}, \boldsymbol{\varepsilon}_{2} \in \mathbb{S}^{d}$, a.e. $(\boldsymbol{x}, t) \in Q$ with $m_{\mathscr{A}}>0$.

(d) $\|\mathscr{A}(\boldsymbol{x}, t, \boldsymbol{\varepsilon})\|_{\mathbb{S}^{d}} \leq \bar{a}_{0}(\boldsymbol{x}, t)+\bar{a}_{1}\|\boldsymbol{\varepsilon}\|_{\mathbb{S}^{d}}$ for all $\boldsymbol{\varepsilon} \in \mathbb{S}^{d}$.

a.e. $(\boldsymbol{x}, t) \in Q$ with $\bar{a}_{0} \in L^{2}(Q), \bar{a}_{0} \geq 0$ and $\bar{a}_{1}>0$.

(e) $\mathscr{A}(\boldsymbol{x}, t, \mathbf{0})=\mathbf{0}$ for a.e. $(\boldsymbol{x}, t) \in Q$.

The elasticity operator $\mathscr{B}: Q \times \mathbb{S}^{d} \rightarrow \mathbb{S}^{d}$ is such that

(a) $\mathscr{B}(\cdot, \cdot, \boldsymbol{\varepsilon})$ is measurable on $Q$ for all $\boldsymbol{\varepsilon} \in \mathbb{S}^{d}$.

(b) $\left\|\mathscr{B}\left(\boldsymbol{x}, t, \boldsymbol{\varepsilon}_{1}\right)-\mathscr{B}\left(\boldsymbol{x}, t, \boldsymbol{\varepsilon}_{2}\right)\right\|_{\mathbb{S}^{d}} \leq L_{\mathscr{B}}\left\|\boldsymbol{\varepsilon}_{1}-\boldsymbol{\varepsilon}_{2}\right\|_{\mathbb{S}^{d}}$

for all $\varepsilon_{1}, \varepsilon_{2} \in \mathbb{S}^{d}$, a.e. $(\boldsymbol{x}, t) \in Q$ with $L_{\mathscr{B}}>0$.

(c) $\mathscr{B}(\cdot, \cdot, \mathbf{0}) \in L^{2}\left(Q ; \mathbb{S}^{d}\right)$.

The relaxation operator $\mathscr{K}: Q \times \mathbb{S}^{d} \rightarrow \mathbb{S}^{d}$ is such that

(a) $\mathscr{K}(\cdot, \cdot, \boldsymbol{\varepsilon})$ is measurable on $Q$ for all $\boldsymbol{\varepsilon} \in \mathbb{S}^{d}$.

(b) $\left\|\mathscr{K}\left(\boldsymbol{x}, t, \boldsymbol{\varepsilon}_{1}\right)-\mathscr{K}\left(\boldsymbol{x}, t, \boldsymbol{\varepsilon}_{2}\right)\right\|_{\mathbb{S}^{d}} \leq L \mathscr{K}\left\|\boldsymbol{\varepsilon}_{1}-\boldsymbol{\varepsilon}_{2}\right\|_{\mathbb{S}^{d}}$

for all $\boldsymbol{\varepsilon}_{1}, \boldsymbol{\varepsilon}_{2} \in \mathbb{S}^{d}$, a.e. $(\boldsymbol{x}, t) \in Q$ with $L_{\mathscr{K}}>0$.

(c) $\mathscr{K}(\cdot, \cdot, \mathbf{0}) \in L^{2}\left(Q ; \mathbb{S}^{d}\right)$.

The stiffness coefficient $k: \Sigma_{C} \times \mathbb{R} \rightarrow \mathbb{R}_{+}$is such that

(a) $k(\cdot, \cdot, r)$ is measurable on $\Sigma_{C}$ for all $r \in \mathbb{R}$.

(b) $\left|k\left(\boldsymbol{x}, t, r_{1}\right)-k\left(\boldsymbol{x}, t, r_{2}\right)\right| \leq L_{k}\left|r_{1}-r_{2}\right|$ for all $r_{1}, r_{2} \in \mathbb{R}$, a.e. $(\boldsymbol{x}, t) \in \Sigma_{C}$ with $L_{k}>0$.

(c) $0 \leq k(\boldsymbol{x}, t, r) \mid \leq \bar{k}$ for all $r \in \mathbb{R}$, a.e. $(\boldsymbol{x}, t) \in \Sigma_{C}$ with $\bar{k}>0$.

The contact function $p: \Sigma_{C} \times \mathbb{R} \rightarrow \mathbb{R}_{+}$is such that

(a) $p(\cdot, \cdot, r)$ is measurable on $\Sigma_{C}$ for all $r \in \mathbb{R}$.

(b) $\left|p\left(\boldsymbol{x}, t, r_{1}\right)-p\left(\boldsymbol{x}, t, r_{2}\right)\right| \leq L_{p}\left|r_{1}-r_{2}\right|$ for all $r_{1}, r_{2} \in \mathbb{R}$, a.e. $(\boldsymbol{x}, t) \in \Sigma_{C}$ with $L_{p}>0$. 

(c) $|p(\boldsymbol{x}, t, r)| \leq \bar{p}$ for all $r \in \mathbb{R}$, a.e. $(\boldsymbol{x}, t) \in \Sigma_{C}$ with $\bar{p}>0$.

The memory function $b: \Sigma_{C} \times \mathbb{R} \rightarrow \mathbb{R}_{+}$is such that

(a) $b(\cdot, \cdot, r)$ is measurable on $\Sigma_{C}$ for all $r \in \mathbb{R}$.

(b) $\left|b\left(\boldsymbol{x}, t, r_{1}\right)-b\left(\boldsymbol{x}, t, r_{2}\right)\right| \leq L_{b}\left|r_{1}-r_{2}\right|$ for all $r_{1}, r_{2} \in \mathbb{R}$, a.e. $(\boldsymbol{x}, t) \in \Sigma_{C}$ with $L_{b}>0$.

(c) $b(\boldsymbol{x}, t, 0) \in L^{2}\left(\Sigma_{C}\right)$ a.e. $(\boldsymbol{x}, t) \in \Sigma_{C}$.

We list the regularity for the densities of body forces and tractions, the initial data, and the coefficient of friction.

$$
\begin{aligned}
& \boldsymbol{f}_{0} \in L^{2}\left(0, T ; L^{2}\left(\Omega ; \mathbb{R}^{d}\right)\right), \quad \boldsymbol{f}_{N} \in L^{2}\left(0, T ; L^{2}\left(\Gamma_{N} ; \mathbb{R}^{d}\right)\right), \quad \boldsymbol{u}_{0}, \boldsymbol{w}_{0} \in V,(30) \\
& \mu \in L^{\infty}\left(\Gamma_{C}\right), \quad \mu(\boldsymbol{x}) \geq 0 \text { a.e. on } \Gamma_{C} .
\end{aligned}
$$

To shorten the notation, let $\boldsymbol{f}:] 0, T\left[\rightarrow V^{*}\right.$ stand for

$$
\langle\boldsymbol{f}(t), \boldsymbol{v}\rangle_{V^{*} \times V}=\left\langle\boldsymbol{f}_{0}(t), \boldsymbol{v}\right\rangle_{L^{2}\left(\Omega ; \mathbb{R}^{d}\right)}+\left\langle\boldsymbol{f}_{N}(t), \boldsymbol{v}\right\rangle_{L^{2}\left(\Gamma_{N} ; \mathbb{R}^{d}\right)}
$$

for all $v \in V$ and a.e. $t \in] 0, T$ [. By a standard procedure, we get the variational formulation of Problem $\mathcal{P}$.

Problem $\mathcal{P}_{V}$. Find a displacement field $\boldsymbol{u}: Q \rightarrow \mathbb{R}^{d}$ and a stress field $\boldsymbol{\sigma}: Q \rightarrow \mathbb{S}^{d}$ such that

$$
\begin{aligned}
\boldsymbol{\sigma}(t)= & \mathscr{A}\left(t, \boldsymbol{\varepsilon}\left(\boldsymbol{u}^{\prime}(t)\right)\right)+\mathscr{B}(t, \boldsymbol{\varepsilon}(\boldsymbol{u}(t))) \\
& \left.+\int_{0}^{t} \mathscr{K}\left(t-s, \boldsymbol{\varepsilon}\left(\boldsymbol{u}^{\prime}(s)\right)\right) \mathrm{d} s \text { for a.e. } t \in\right] 0, T[ \\
\left\langle\boldsymbol{u}^{\prime \prime}(t), \boldsymbol{v}-\boldsymbol{u}^{\prime}(t)\right\rangle_{V^{*} \times V}+\left(\boldsymbol{\sigma}(t), \boldsymbol{\varepsilon}(\boldsymbol{v})-\boldsymbol{\varepsilon}\left(\boldsymbol{u}^{\prime}(t)\right)\right) \mathcal{H} & \\
& +\int_{\Gamma_{C}}\left(k\left(\int_{0}^{t} u_{v}(s) \mathrm{d} s\right) p\left(t, u_{v}(t)\right)+\int_{0}^{t} b\left(t-s, u_{v}(s)\right) \mathrm{d} s\right) \\
& \left(v_{v}-u_{v}^{\prime}(t)\right) \mathrm{d} \Gamma \\
& +\int_{\Gamma_{C}}\left(\mu \left(k\left(\int_{0}^{t} u_{v}(s) \mathrm{d} s\right) p\left(t, u_{v}(t)\right)\right.\right. \\
& \left.\left.+\int_{0}^{t} b\left(t-s, u_{v}(s)\right) \mathrm{d} s\right)\right)\left(\left\|\boldsymbol{v}_{\tau}\right\|_{\mathbb{R}^{d}}-\left\|\boldsymbol{u}_{\tau}^{\prime}(t)\right\|_{\mathbb{R}^{d}}\right) \mathrm{d} \Gamma \\
\geq\langle & \left.\boldsymbol{f}(t), \boldsymbol{v}\rangle_{V^{*} \times V} \text { for a.e. } t \in\right] 0, T[, \\
\boldsymbol{u}(0)= & \boldsymbol{u}_{0}, \quad \boldsymbol{u}^{\prime}(0)=\boldsymbol{w}_{0} .
\end{aligned}
$$

Now, we comment on the equivalent formulations of Problem $\mathcal{P}_{V}$. First, we denote $\boldsymbol{w}=\boldsymbol{u}^{\prime}$, i.e.,

$$
\boldsymbol{u}(t)=\int_{0}^{t} \boldsymbol{w}(s) \mathrm{d} s+\boldsymbol{u}_{0} \text { for all } t \in[0, T]
$$


Using this notation and inserting (33) into (34), we obtain the following formulation.

Problem $\mathcal{P}_{1}$. Find a velocity field $\boldsymbol{w}: Q \rightarrow \mathbb{R}^{d}$ such that

$$
\begin{aligned}
& \left\langle\boldsymbol{w}^{\prime}(t), \boldsymbol{v}-\boldsymbol{w}(t)\right\rangle_{V^{*} \times V}+(\mathscr{A}(t, \boldsymbol{\varepsilon}(\boldsymbol{w}(t))), \boldsymbol{\varepsilon}(\boldsymbol{v})-\boldsymbol{\varepsilon}(\boldsymbol{w}(t)))_{\mathcal{H}} \\
& \quad+\left(\mathscr{B}\left(t, \boldsymbol{\varepsilon}\left(\int_{0}^{t} \boldsymbol{w}(s) \mathrm{d} s+\boldsymbol{u}_{0}\right)\right)+\int_{0}^{t} \mathscr{K}(t-s, \boldsymbol{\varepsilon}(\boldsymbol{w}(s))) \mathrm{d} s, \boldsymbol{\varepsilon}(\boldsymbol{v})-\boldsymbol{\varepsilon}\left(\boldsymbol{u}^{\prime}(t)\right)\right)_{\mathcal{H}} \\
& \quad+\left(k\left(\int_{0}^{t}\left(\int_{0}^{s} \boldsymbol{w}(r) \mathrm{d} r+\boldsymbol{u}_{0}\right)_{v} \mathrm{~d} s\right) p\left(t,\left(\int_{0}^{s} \boldsymbol{w}(r) \mathrm{d} r+\boldsymbol{u}_{0}\right)_{v}\right)_{v}\right. \\
& \left.\quad+\int_{0}^{t} b\left(t-s,\left(\int_{0}^{s} \boldsymbol{w}(r) \mathrm{d} r+\boldsymbol{u}_{0}\right)_{v}\right) \mathrm{d} s, v_{v}-u_{v}^{\prime}(t)\right)_{L^{2}\left(\Gamma_{C}\right)} \\
& \quad+\left(\mu \left(k\left(\int_{0}^{t}\left(\int_{0}^{s} \boldsymbol{w}(r) \mathrm{d} r+\boldsymbol{u}_{0}\right)_{v} \mathrm{~d} s\right) p\left(t,\left(\int_{0}^{s} \boldsymbol{w}(r) \mathrm{d} r+\boldsymbol{u}_{0}\right)_{v}\right)\right.\right. \\
& \left.\left.\left.\quad+\int_{0}^{t} b\left(t-s,\left(\int_{0}^{s} \boldsymbol{w}(r) \mathrm{d} r+\boldsymbol{u}_{0}\right)_{v}\right) \mathrm{d} s\right)\right),\left\|\boldsymbol{v}_{\tau}\right\|_{\mathbb{R}^{d}}-\left\|\boldsymbol{u}_{\tau}^{\prime}(t)\right\|_{\mathbb{R}^{d}}\right)_{L^{2}\left(\Gamma_{C}\right)} \\
& \left.\geq\langle\boldsymbol{f}(t), \boldsymbol{v}\rangle_{V^{*} \times V} \text { for all } \boldsymbol{v} \in V, \text { a.e. } t \in\right] 0, T[, \\
& \quad \boldsymbol{w}(0)=\boldsymbol{w}_{0} .
\end{aligned}
$$

Obviously, Problems $\mathcal{P}_{V}$ and $\mathcal{P}_{1}$ are equivalent. Indeed, if $(\boldsymbol{u}, \boldsymbol{\sigma})$ is a solution to Problem $\mathcal{P}_{V}$, then $\boldsymbol{w}=\boldsymbol{u}^{\prime}$ solves Problem $\mathcal{P}_{1}$. If $\boldsymbol{w}$ satisfies Problem $\mathcal{P}_{1}$, then we find a displacement $\boldsymbol{u}$, while the stress field in Problem $\mathcal{P}_{V}$ can be uniquely determined by (33).

Next, we pass to other formulations of Problem $\mathcal{P}_{1}$, in terms of the velocity. To this end, let $Y=L^{2}\left(\Gamma_{C}\right), U=L^{2}\left(\Gamma_{C} ; \mathbb{R}^{d}\right)$, and define the following operators $A:] 0, T\left[\times V \rightarrow V^{*}, R: \mathcal{V} \rightarrow L^{2}(0, T ; Y), R_{1}, S: \mathcal{V} \rightarrow \mathcal{V}^{*}, N: V \rightarrow U\right.$ and functional $\varphi: Y \times U \rightarrow \mathbb{R}$, respectively, by

$$
\begin{aligned}
& \left.\langle A(t, \boldsymbol{u}), \boldsymbol{v}\rangle_{V^{*} \times V}=(\mathscr{A}(t, \boldsymbol{\varepsilon}(\boldsymbol{u})), \boldsymbol{\varepsilon}(\boldsymbol{v}))_{\mathcal{H}} \text { for all } \boldsymbol{u}, \boldsymbol{v} \in V, \text { a.e. } t \in\right] 0, T \\
& \left.(S \boldsymbol{w})(t)=\int_{0}^{t} \boldsymbol{w}(s) \mathrm{d} s+\boldsymbol{u}_{0} \text { for all } \boldsymbol{w} \in \mathcal{V} \text {, a.e. } t \in\right] 0, T[ \\
& (R \boldsymbol{w})(t)=k\left(\int_{0}^{t}(S \boldsymbol{w})_{v}(s) \mathrm{d} s\right) p\left(t,(S \boldsymbol{w})_{v}(t)\right)+\int_{0}^{t} b\left(t-s,(S \boldsymbol{w})_{\mathcal{V}}(s)\right) \mathrm{d} s \\
& \quad \text { for all } \boldsymbol{w} \in \mathcal{V} \text {, a.e. } t \in] 0, T[ \\
& \left(R_{1} \boldsymbol{w}\right)(t)=\mathscr{B}(t, \boldsymbol{\varepsilon}(S \boldsymbol{w})(t))+\int_{0}^{t} \mathscr{K}(t-s, \boldsymbol{\varepsilon}(\boldsymbol{w}(s))) \mathrm{d} s \\
& \quad \text { for all } \boldsymbol{w} \in \mathcal{V}, \text { a.e. } t \in] 0, T[, \\
& N=\gamma: V \rightarrow U \text { is the trace operator, } \\
& \varphi(y, \boldsymbol{z})=\left(y, z_{v}\right)_{L^{2}\left(\Gamma_{C}\right)}+\left(\mu y,\left\|\boldsymbol{z}_{\tau}\right\|_{\left.\mathbb{R}^{d}\right)_{L^{2}\left(\Gamma_{C}\right)} \quad \text { for all } y \in Y, \boldsymbol{z} \in U \text {. }}\right.
\end{aligned}
$$

Under this notation, we consider the following history-dependent variational inequality and the evolution inclusion. 
Problem $\mathcal{P}_{2}$. Find $w \in \mathcal{W}$ such that

$$
\begin{aligned}
& \left\langle\boldsymbol{w}^{\prime}(t)+A(t, \boldsymbol{w}(t))+\left(R_{1} \boldsymbol{w}\right)(t)-\boldsymbol{f}(t), \boldsymbol{v}-\boldsymbol{w}(t)\right\rangle_{V^{*} \times V} \\
& \quad+\varphi((R \boldsymbol{w})(t), N \boldsymbol{v})-\varphi((R \boldsymbol{w})(t), N \boldsymbol{w}(t)) \geq 0 \text { for all } \boldsymbol{v} \in V, \text { a.e. } t, \\
& \boldsymbol{w}(0)=\boldsymbol{w}_{0}
\end{aligned}
$$

Problem $\mathcal{P}_{3}$. Find $w \in \mathcal{W}$ such that

$$
\begin{aligned}
& \boldsymbol{w}^{\prime}(t)+A(t, \boldsymbol{w}(t))+\left(R_{1} \boldsymbol{w}\right)(t)+N^{*} \partial \varphi(t,(R \boldsymbol{w})(t), N \boldsymbol{w}(t)) \ni \boldsymbol{f}(t) \text { a.e. } t \\
& \boldsymbol{w}(0)=\boldsymbol{w}_{0}
\end{aligned}
$$

Theorem 6.1 Under the hypotheses (24)-(31), Problem $\mathcal{P}_{3}$ has the unique solution. Moreover, Problems $\mathcal{P}_{1}, \mathcal{P}_{2}$, and $\mathcal{P}_{3}$ are equivalent.

Proof For the proof of existence and uniqueness, we will verify the hypotheses of Theorem 3.2.

It is clear that under (24), the operator $A$ defined by (36) satisfies $H(A)$ with $m_{A}=m_{\mathscr{A}}, a_{0}(t)=\sqrt{2}\left\|\bar{a}_{0}(t)\right\|_{L^{2}(\Omega)}$, and $a_{1}=\sqrt{2} \bar{a}_{1}$, see [18, Theorem 14.2]. Using (31), the function $\varphi$, given by (41), satisfies $H(\varphi)$ with $c_{0 \varphi}=c_{2 \varphi}=0$ and $c_{1 \varphi}=\beta_{\varphi}=1+\|\mu\|_{L^{\infty}\left(\Gamma_{C}\right)}$, see the proof in [32, Theorem 7].

Next, obviously, the operator $N$ defined by (40) satisfies $H(N)$. Because of (30), function $f$ given by (32) satisfies $\left(H_{0}\right)$. Subsequently, from the proof of [32, Theorem 7] and (27)-(29), it follows that operator $R$ defined by (38) satisfies

$$
\left\|\left(R \boldsymbol{w}_{1}\right)(t)-\left(R \boldsymbol{w}_{2}\right)(t)\right\|_{Y} \leq c \int_{0}^{t}\left\|\boldsymbol{w}_{1}(s)-\boldsymbol{w}_{2}(s)\right\|_{L^{2}\left(\Gamma_{C} ; \mathbb{R}^{d}\right)} \mathrm{d} s
$$

for all $\boldsymbol{w}_{1}, \boldsymbol{w}_{2} \in \mathcal{V}$, a.e. $\left.t \in\right] 0, T\left[\right.$ with $c_{0}=\bar{k} L_{p}+\left(\bar{p} L_{k}+L_{b}\right) T$. This implies that the condition $\left(H_{2}\right)(\mathrm{i})$ holds with $c_{R}=c_{0}\|\gamma\|$ and, moreover,

$$
\|(R \mathbf{0} \mathcal{V})(t)\|_{Y} \leq \bar{k} \bar{p}+\int_{0}^{t}\left\|b_{0}(s)\right\|_{L^{2}\left(\Gamma_{C}\right)} \mathrm{d} s
$$

with $b_{0}(x, t)=\|b(x, t, 0)\|_{\mathbb{R}^{d}}$ and $b_{0} \in L^{2}\left(\Sigma_{C}\right)$, a.e. $\left.t \in\right] 0, T$ [. Further, exploiting (25) and (26), we know that the operator $R_{1}$, given by (39), satisfies the condition $\left(H_{2}\right)$ (ii) with $c_{R 1}=L_{\mathscr{B}}+L_{\mathscr{K}}$, and

$$
\left\|\left(R_{1} \mathbf{0}_{\mathcal{V}}\right)(t)\right\|_{V^{*}} \leq\left\|b_{1}(t)\right\|_{L^{2}(\Omega)}+\int_{0}^{t}\left\|k_{1}(s)\right\|_{L^{2}(\Omega)} \mathrm{d} s
$$

with $b_{1}(x, t)=\|\mathscr{B}(\boldsymbol{x}, t, \mathbf{0})\|_{\mathbb{S}^{d}}$ and $k_{1}(x, t)=\|\mathscr{K}(\boldsymbol{x}, t, \mathbf{0})\|_{\mathbb{S}^{d}}, b_{1}, k_{1} \in L^{2}(Q)$. In conclusion, $R$ and $R_{1}$ are history-dependent operators. Having verified hypotheses $H(A), H(\varphi), H(N),\left(H_{0}\right)$, and $\left(H_{2}\right)$, we are now in a position to apply Theorem 3.2 to deduce that Problem $\mathcal{P}_{3}$ has a unique solution. 
Next, by the definition of the subdifferential, it is easy to see that the solution to Problem $\mathcal{P}_{3}$ solves also Problem $\mathcal{P}_{2}$. Further, using our notation, we can see that every solution to Problem $\mathcal{P}_{2}$ is a solution to Problem $\mathcal{P}_{1}$. Finally, by a direct calculation, we show the uniqueness of solution to Problem $\mathcal{P}_{1}$. This completes the proof.

From Theorem 6.1, we deduce our existence and uniqueness result for Problem $\mathcal{P}_{V}$.

Corollary 6.1 Under the hypotheses (24)-(31), Problem $\mathcal{P}_{V}$ has the unique solution such that $\boldsymbol{u} \in W^{1,2}(0, T ; V), \boldsymbol{\sigma} \in L^{2}(0, T ; \mathcal{H})$, Div $\boldsymbol{\sigma} \in L^{2}\left(0, T ; V^{*}\right)$.

Note that Corollary 6.1 was first proved in [32, Theorem 7] under the additional smallness condition of the form $m_{\mathcal{A}}>2 \sqrt{2}\left(1+\|\mu\|_{L^{\infty}\left(\Gamma_{C}\right)}\right)\|\gamma\|^{2}$, which is relaxed now.

Consider the following nonlinear optimal control problem that contains distributed, boundary and initial condition controls, and the cost functional that combines observations within the domain, on the boundary, and at the terminal time.

$$
\begin{aligned}
& \min J\left(\boldsymbol{w}, \boldsymbol{f}_{0}, \boldsymbol{f}_{N}, \boldsymbol{w}_{0}\right)=\rho_{1}\left\|\boldsymbol{w}_{0}\right\|^{2}+\int_{\Omega} \rho_{2}\left|\boldsymbol{w}(x, T)-\boldsymbol{w}_{T}(x)\right|^{2} \mathrm{~d} x \\
& +\int_{Q} \rho_{3}\left|\boldsymbol{w}(x, t)-\boldsymbol{w}_{d}(x, t)\right|^{2} \mathrm{~d} x \mathrm{~d} t+\int_{Q} \rho_{4}\left|\boldsymbol{f}_{0}(x, t)\right|^{2} \mathrm{~d} x \mathrm{~d} t \\
& +\int_{\Sigma_{N}} \rho_{5}\left|\boldsymbol{f}_{N}(x, t)\right|^{2} \mathrm{~d} \Gamma \mathrm{d} t
\end{aligned}
$$

subject to

$$
\begin{aligned}
& \boldsymbol{w}^{\prime}(t)+A \boldsymbol{w}(t)+\left(R_{1} \boldsymbol{w}\right)(t)+N^{*} \partial \varphi(t,(R \boldsymbol{w})(t), N \boldsymbol{w}(t)) \ni \boldsymbol{g}(t) \text { a.e. } t \\
& \boldsymbol{w}(0)=\boldsymbol{w}_{0}
\end{aligned}
$$

with $\langle\boldsymbol{g}(t), \boldsymbol{v}\rangle_{V^{*} \times V}=\langle\tilde{\boldsymbol{f}}(t), \boldsymbol{v}\rangle_{V^{*} \times V}+\left\langle\boldsymbol{f}_{0}(t), \boldsymbol{v}\right\rangle_{H}+\left\langle\boldsymbol{f}_{N}(t), \boldsymbol{v}\right\rangle_{L^{2}\left(\Gamma_{N} ; \mathbb{R}^{d}\right)}$ for $\boldsymbol{v} \in V$, a.e. $t \in] 0, T[$, and

$$
\begin{aligned}
& \left.\left\|\boldsymbol{f}_{0}(t)\right\|_{H} \leq r_{1}(t), \quad\left\|\boldsymbol{f}_{N}(t)\right\|_{L^{2}\left(\Gamma_{N} ; \mathbb{R}^{d}\right)} \leq r_{2}(t) \text { a.e. } t \in\right] 0, T[ \\
& \boldsymbol{f}_{0}(\cdot, \cdot), \boldsymbol{f}_{N}(\cdot, \cdot) \text { aremeasurable, }\left\|\boldsymbol{w}_{0}\right\|_{V} \leq r
\end{aligned}
$$

In problem (46), the operator $A: V \rightarrow V^{*}$ is given by

$$
\langle A \boldsymbol{w}, \boldsymbol{v}\rangle_{V^{*} \times V}=(\mathscr{A} \boldsymbol{\varepsilon}(\boldsymbol{w}), \boldsymbol{\varepsilon}(\boldsymbol{v}))_{\mathcal{H}} \text { for all } \boldsymbol{w}, \boldsymbol{v} \in V
$$

and the data $R, R_{1}, N$, and $\varphi$ are defined by (36)-(41) with, recall, $Y=L^{2}\left(\Gamma_{C}\right)$ and $U=L^{2}\left(\Gamma_{C} ; \mathbb{R}^{d}\right)$. The state $\boldsymbol{w} \in \mathcal{W}$ of system (46) is associated with the constrained control $\left(\boldsymbol{f}_{0}, \boldsymbol{f}_{N}, \boldsymbol{w}_{0}\right) \in L^{2}(0, T ; X) \times V$, where we set $X=H \times L^{2}\left(\Gamma_{N} ; \mathbb{R}^{d}\right)$.

We need the following hypotheses on the data of problem (45)-(47).

$\underline{H(\mathscr{A})_{1}}: \quad \mathscr{A}: \Omega \times \mathbb{S}^{d} \rightarrow \mathbb{S}^{d}$ is such that

(i) $\mathscr{A}(\boldsymbol{x}, \boldsymbol{\varepsilon})=a(\boldsymbol{x}) \boldsymbol{\varepsilon}$ for all $\boldsymbol{\varepsilon} \in \mathbb{S}^{d}$, a.e. $\boldsymbol{x} \in \Omega$. 
(ii) $a(\boldsymbol{x})=\left\{a_{i j k l}(\boldsymbol{x})\right\}, a_{i j k l}=a_{j i k l}=a_{l k i j} \in L^{\infty}(\Omega), i, j, k, l=1, \ldots, d$.

(iii) $a_{i j k l}(\boldsymbol{x}) \varepsilon_{i j} \varepsilon_{k l} \geq \alpha\|\boldsymbol{\varepsilon}\|^{2}$ for all $\boldsymbol{\varepsilon}=\left(\boldsymbol{\varepsilon}_{i j}\right) \in \mathbb{S}^{d}$, a.e. $\boldsymbol{x} \in \Omega$ with $\alpha>0$.

$\underline{H(\mathscr{B})_{1}}: \quad \mathscr{B}: \Omega \times \mathbb{S}^{d} \rightarrow \mathbb{S}^{d}$ is such that

(i) $\mathscr{B}(\boldsymbol{x}, \boldsymbol{\varepsilon})=b(\boldsymbol{x}) \boldsymbol{\varepsilon}$ for all $\boldsymbol{\varepsilon} \in \mathbb{S}^{d}$, a.e. $\boldsymbol{x} \in \Omega$.

(ii) $b(\boldsymbol{x})=\left\{b_{i j k l}(\boldsymbol{x})\right\}, b_{i j k l}=b_{j i k l}=b_{l k i j} \in L^{\infty}(\Omega), i, j, k, l=1, \ldots, d$.

(iii) $b_{i j k l}(\boldsymbol{x}) \varepsilon_{i j} \varepsilon_{k l} \geq 0$ for all $\boldsymbol{\varepsilon}=\left(\boldsymbol{\varepsilon}_{i j}\right) \in \mathbb{S}^{d}$, a.e. $\boldsymbol{x} \in \Omega$.

$\underline{H(\mathscr{K})_{1}}: \quad \mathscr{K}: Q \times \mathbb{S}^{d} \rightarrow \mathbb{S}^{d}$ is such that

(i) $\mathscr{K}(\boldsymbol{x}, t, \boldsymbol{\varepsilon})=\kappa(\boldsymbol{x}, t) \boldsymbol{\varepsilon}$ for all $\boldsymbol{\varepsilon} \in \mathbb{S}^{d}$, a.e. $(\boldsymbol{x}, t) \in Q$.

(ii) $\kappa(\boldsymbol{x}, t)=\left\{\kappa_{i j k l}(\boldsymbol{x}, t)\right\}, \kappa_{i j k l}=\kappa_{j i k l}=\kappa_{l k i j} \in L^{\infty}(Q), i, j, k, l=1, \ldots, d$.

$\left(H_{5}\right): \quad \rho_{1}, \rho_{2} \in L^{\infty}(\Omega), \rho_{3}, \rho_{4} \in L^{\infty}(Q), \rho_{5} \in L^{\infty}\left(\Sigma_{N}\right), \rho_{i}$ are nonnegative weights for $i=1, \ldots, 5, r_{1}, r_{2} \in L^{\infty}(0, T), r>0, \widetilde{f} \in \mathcal{V}^{*}, w_{T} \in H$, and $\boldsymbol{w}_{d} \in L^{2}(Q)$.

Theorem 6.2 Under the hypotheses $H(\mathscr{A})_{1}, H(\mathscr{B})_{1}, H(\mathscr{K})_{1},\left(H_{5}\right)$, and (27)-(31), the optimal control problem (45)-(47) has an optimal solution $\left(\boldsymbol{w}, \boldsymbol{f}_{0}, \boldsymbol{f}_{N}, \boldsymbol{w}_{0}\right) \in$ $\mathcal{W} \times L^{2}(0, T ; X) \times V$.

Proof We will apply Theorem 5.1, and to this end, we verify its hypotheses. Recall that we work in the evolution triple of spaces with $V$ and $H$ defined in (23).

First, under $H(\mathscr{A})_{1}, A$ defined by (48) clearly satisfies $A \in \mathcal{L}\left(V, V^{*}\right)$, and $H(A)_{1}$ with $a_{0}=0, a_{1}=\|A\|_{\mathcal{L}\left(V, V^{*}\right)}$ and $m_{A}=\alpha$. By [29, Theorem 2.18], we know that the Nemytskii operator $\mathcal{N}: \mathcal{W} \subset \mathcal{V} \rightarrow L^{2}(0, T ; U)$ corresponding to $N$ is compact. Therefore, the condition $H(N)_{1}$ holds.

Second, we verify the condition $\left(H_{4}\right)$ (i)-(iii). We prove the compactness of the operator $R$. Let $\boldsymbol{v}_{n} \rightarrow \boldsymbol{v}$ weakly in $\mathcal{W}$. From [29, Theorem 2.18], we know that $\gamma \boldsymbol{v}_{n} \rightarrow \gamma \boldsymbol{v}$ in $L^{2}\left(0, T ; L^{2}\left(\Gamma_{C} ; \mathbb{R}^{d}\right)\right)$, where $\gamma: \mathcal{V} \rightarrow L^{2}\left(0, T ; L^{2}\left(\Gamma_{C} ; \mathbb{R}^{d}\right)\right)$ is the Nemytskii operator corresponding to the trace $\gamma$ (for simplicity denoted in the same way). By (42) and Hölder's inequality, we have

$$
\begin{aligned}
\left\|\left(R \boldsymbol{v}_{n}\right)(t)-(R \boldsymbol{v})(t)\right\|_{Y} & \leq c \int_{0}^{t}\left\|\boldsymbol{v}_{n}(r)-\boldsymbol{v}(r)\right\|_{L^{2}\left(\Gamma_{C} ; \mathbb{R}^{d}\right)} \mathrm{d} r \\
& \leq c \sqrt{T}\left\|\boldsymbol{v}_{n}-\boldsymbol{v}\right\|_{L^{2}\left(0, T ; L^{2}\left(\Gamma_{C} ; \mathbb{R}^{d}\right)\right)}
\end{aligned}
$$

for a.e. $t \in] 0, T$ [ with $c>0$. Hence, it follows that $\left(R \boldsymbol{v}_{n}\right)(t) \rightarrow(R \boldsymbol{v})(t)$ in $Y$, for a.e. $t \in] 0, T$ [. By the Lebesgue dominated convergence theorem, we get $R \boldsymbol{v}_{n} \rightarrow R \boldsymbol{v}$ in $L^{2}(0, T ; Y)$. Thus, the operator $R: \mathcal{W} \subset \mathcal{V} \rightarrow L^{2}(0, T ; Y)$ is compact.

To prove the continuity of $\mathcal{R}_{1}$ in weak topologies, we set

$$
\begin{aligned}
& \left\langle\left(R_{11} \boldsymbol{w}\right)(t), \boldsymbol{v}\right\rangle_{V^{*} \times V}=\left(\mathscr{B}\left(\int_{0}^{t} \boldsymbol{\varepsilon}(\boldsymbol{w}(s)) \mathrm{d} s+\boldsymbol{u}_{0}\right), \boldsymbol{\varepsilon}(\boldsymbol{v})\right)_{\mathcal{H}}, \\
& \left\langle\left(R_{12} \boldsymbol{w}\right)(t), \boldsymbol{v}\right\rangle_{V^{*} \times V}=\left(\int_{0}^{t} \mathscr{K}(t-s) \boldsymbol{\varepsilon}(\boldsymbol{w}(s)) \mathrm{d} s, \boldsymbol{\varepsilon}(\boldsymbol{v})\right)_{\mathcal{H}}
\end{aligned}
$$


for all $\boldsymbol{w} \in \mathcal{V}, \boldsymbol{v} \in V, t \in] 0, T$. Let $\left\{\boldsymbol{v}_{n}\right\} \subset \mathcal{V}$ be such that $\boldsymbol{v}_{n} \rightarrow \boldsymbol{v}$ weakly in $\mathcal{V}$. Then, for all $\chi \in V^{*}$, all $t \in[0, T]$, we have

$$
\begin{aligned}
& \left\langle\int_{0}^{t} \boldsymbol{v}_{n}(s) \mathrm{d} s, \chi\right\rangle_{V^{*} \times V}=\int_{0}^{t}\left\langle\boldsymbol{v}_{n}(s), \chi\right\rangle_{V^{*} \times V} \mathrm{~d} s=\left\langle\boldsymbol{v}_{n}, \chi\right\rangle_{\mathcal{V}^{*} \times \mathcal{V}} \\
& \rightarrow\langle\boldsymbol{v}, \chi\rangle_{\mathcal{V}^{*} \times \mathcal{V}}=\int_{0}^{t}\langle\boldsymbol{v}(s), \chi\rangle_{V^{*} \times V} \mathrm{~d} s=\left\langle\int_{0}^{t} \boldsymbol{v}(s) \mathrm{d} s, \chi\right\rangle_{V^{*} \times V},
\end{aligned}
$$

that is,

$$
\int_{0}^{t} \boldsymbol{v}_{n}(s) \mathrm{d} s+\boldsymbol{u}_{0} \rightarrow \int_{0}^{t} \boldsymbol{v}(s) \mathrm{d} s+\boldsymbol{u}_{0} \text { weakly in } V, \text { for all } t \in[0, T]
$$

Since $\mathscr{B}$ is linear and continuous, we deduce that $R_{11} \boldsymbol{v}_{n} \rightarrow R_{11} \boldsymbol{v}$ weakly in $\mathcal{V}^{*}$. Also, since $R_{12}$ is linear and continuous, it is also weakly-weakly continuous, and hence, $R_{12} \boldsymbol{v}_{n} \rightarrow R_{12} \boldsymbol{v}$ weakly in $\mathcal{V}^{*}$. We infer that $R_{1}$ is weakly-weakly continuous. Further, from (43) and (38), it is easily seen that $\left(R \mathbf{0}_{\mathcal{V}}, R_{1} \mathbf{0}_{\mathcal{V}}\right)$ belongs to a bounded subset of $L^{2}\left(0, T ; Y \times V^{*}\right)$. Hence, $\left(H_{4}\right)$ is verified.

Third, let $B: X \rightarrow V^{*}$ be defined by

$$
\left\langle B\left(z_{1}, z_{2}\right), \boldsymbol{v}\right\rangle_{V^{*} \times V}=\left\langle z_{1}, i \boldsymbol{v}\right\rangle_{H}+\left\langle z_{2}, \gamma \boldsymbol{v}\right\rangle_{L^{2}\left(\Gamma_{N} ; \mathbb{R}^{d}\right)}
$$

for $\left(z_{1}, z_{2}\right) \in X, v \in V$, where $i: V \rightarrow H$ is the embedding operator. It is obvious that $B$ is a linear and continuous operator. Let $U:] 0, T\left[\rightrightarrows 2^{X} \backslash\{\emptyset\}\right.$ be given by

$$
U(t)=\left\{z_{1} \in H:\left\|z_{1}\right\|_{H} \leq r_{1}(t)\right\} \times\left\{z_{2} \in L^{2}\left(\Gamma_{N} ; \mathbb{R}^{d}\right):\left\|z_{1}\right\|_{L^{2}\left(\Gamma_{N} ; \mathbb{R}^{d}\right)} \leq r_{2}(t)\right\}
$$

for a.e. $t \in] 0, T$ [ and $V_{a d}$ be the closed ball in $V$ centered at zero and radius $r>0$. By the hypotheses, we deduce that $H(B), H\left(V_{a d}\right)$, and $H\left(U_{a d}\right)$ are satisfied.

Finally, we consider the components of the cost functional which are defined by $l_{1}(\boldsymbol{v})=\rho_{1}\|\boldsymbol{v}\|^{2}$ for $\boldsymbol{v} \in V, l_{2}(\boldsymbol{v})=\int_{\Omega} \rho_{2}\left|\boldsymbol{v}-\boldsymbol{w}_{T}\right|^{2} \mathrm{~d} x$ for $\boldsymbol{v} \in H$, and

$$
L\left(t, \boldsymbol{w}, \boldsymbol{v}_{1}, \boldsymbol{v}_{2}\right)=\int_{\Omega} \rho_{3}\left|\boldsymbol{w}-\boldsymbol{w}_{d}\right|^{2} \mathrm{~d} x+\int_{\Omega} \rho_{4}\left|\boldsymbol{v}_{1}\right|^{2} \mathrm{~d} x+\int_{\Gamma_{N}} \rho_{5}\left|\boldsymbol{v}_{2}\right|^{2} \mathrm{~d} \Gamma
$$

for $\boldsymbol{w} \in H,\left(\boldsymbol{v}_{1}, \boldsymbol{v}_{2}\right) \in X$, a.e. $\left.t \in\right] 0, T$. It is easy to see, using the weak lower semicontinuity of the norm, that these functionals satisfy $H\left(l_{1}, l_{2}\right)$ and $H(L)$. Furthermore, the hypothesis $H$ (feas) is evidently satisfied.

Under the above notation, we can rewrite problem (45)-(47) in the abstract form (22) and invoke Theorem 5.1. We conclude that the optimal control problem (45)-(47) has at least one optimal solution. The proof is complete. 


\section{Conclusions}

We have studied the optimal control problem for the weak formulation of the contact problem in terms of the velocity. Alternatively, following the technique of this paper, it is possible to obtain results for the optimal control problem for the variational inequality formulated in terms of the displacement.

The formulation and hypotheses on the optimal control problem (22) are quite general, and they allow one to incorporate other types of cost functionals, whose interpretation in contact mechanics is very important. The cost in (45) measures the deviation from a given state and fulfills a minimum expenditure condition. It permits to assign the relative importance of each criterion with respect to the other by an appropriate selection of the weight coefficients.

It would be of significant interest to extend our results to evolution variationalhemivariational inequalities, which are applicable to other dynamic contact problems in mechanics. Furthermore, it would be desirable to study problems of practical importance. They include a time optimal control problem, a maximum stay control problem, problems with controls in the potential, and minimax problems, in which one tries to minimize the maximum cost. It is also natural to explore optimal control for problems of contact mechanics, when the state of the system is not uniquely determined.

Moreover, the systems under consideration in this paper cover particular cases of evolution inclusions governed by various types of hereditary and delay-differential inclusions, differential-algebraic inclusions with time delays, functional-differential inclusions of neutral type, etc. Optimal control problems, for all the aforementioned history-dependent evolution inclusions, were also studied in detail in [44, Chapter 7]. Finally, it would be interesting to study classes of subdifferential evolution inclusions, which model the so-called sweeping/Moreau processes. Optimal control problems for sweeping processes have been formulated quite recently, and they occur to be highly challenging mathematically; see [45-47].

Acknowledgements This project has received funding from the European Union's Horizon 2020 Research and Innovation Programme under the Marie Skłodowska-Curie Grant Agreement No. 823731 CONMECH. It is supported by Natural Science Foundation of Guangxi (Grant No. 2018GXNSFAA281353), Beibu Gulf University Project No. 2018KYQD06, and the projects financed by the Ministry of Science and Higher Education of Republic of Poland under Grants Nos. 4004/GGPJII/H2020/2018/0 and 440328/PnH2/2019.

Open Access This article is licensed under a Creative Commons Attribution 4.0 International License, which permits use, sharing, adaptation, distribution and reproduction in any medium or format, as long as you give appropriate credit to the original author(s) and the source, provide a link to the Creative Commons licence, and indicate if changes were made. The images or other third party material in this article are included in the article's Creative Commons licence, unless indicated otherwise in a credit line to the material. If material is not included in the article's Creative Commons licence and your intended use is not permitted by statutory regulation or exceeds the permitted use, you will need to obtain permission directly from the copyright holder. To view a copy of this licence, visit http://creativecommons.org/licenses/by/4.0/.

\section{References}

1. Lions, J.-L.: Optimal Control of Systems Governed by Partial Differential Equations. Springer, Heidelberg (1971) 
2. Tröltzsch, F.: Optimal Control of Partial Differential Equations: Theory, Methods and Applications. Graduate Studies in Mathematics, vol. 112. AMS, Providence (2010)

3. Ahmed, N.U., Teo, K.L.: Optimal Control of Distributed Parameters Systems. Elsevier North Holland, New York (1981)

4. Barbu, V.: Optimal Control of Variational Inequalities. Pitman, London (1984)

5. Tiba, D.: Optimal Control of Nonsmooth Distributed Parameter Systems. Lecture Notes in Mathematics, vol. 1459. Springer, Berlin (1990)

6. Hu, S., Papageorgiou, N.S.: Time-Dependent Subdifferential Evolution Inclusions and Optimal Control, vol. 632. Memoirs of the American Mathematical Society, Providence (1998)

7. Hu, S., Papageorgiou, N.S.: Handbook of Multivalued Analysis, Volume II: Applications. Kluwer, Dordrecht (2000)

8. Mordukhovich, B.S.: Variational analysis of evolution inclusions. SIAM J. Optim. 18, 752-777 (2007)

9. Papageorgiou, N.S.: Extremal solutions of evolution inclusions associated with time dependent convex subdifferentials. Math. Nachr. 158, 219-232 (1992)

10. Papageorgiou, N.S.: On parametric evolution inclusions of the subdifferential type with applications to optimal control problems. Trans. Am. Math. Soc. 347, 203-231 (1995)

11. Tolstonogov, A.A.: Relaxation in nonconvex optimal control problems with subdifferential operators. J. Math. Sci. 140, 850-872 (2007)

12. Migórski, S.: Existence of solutions for a class of history-dependent evolution hemivariational inequalities. Dyn. Syst. Appl. 21, 319-330 (2012)

13. Migórski, S., Ochal, A., Sofonea, M.: Integrodifferential hemivariational inequalities with applications to viscoelastic frictional contact. Math. Models Methods Appl. Sci. 18, 271-290 (2008)

14. Migórski, S., Ochal, A., Sofonea, M.: History-dependent subdifferential inclusions and hemivariational inequalities in contact mechanics. Nonlinear Anal. RWA 12, 3384-3396 (2011)

15. Migórski, S., Ochal, A., Sofonea, M.: History-dependent variational-hemivariational inequalities in contact mechanics. Nonlinear Anal. RWA 22, 604-618 (2015)

16. Sofonea, M., Han, W., Migórski, S.: Numerical analysis of history-dependent variationalhemivariational inequalities with applications to contact problems. Eur. J. Appl. Math. 26, 427-452 (2015)

17. Sofonea, M., Matei, A.: History-dependent quasivariational inequalities arising in contact mechanics. Eur. J. Appl. Math. 22, 471-491 (2011)

18. Sofonea, M., Migórski, S., Ochal, A.: Two history-dependent contact problems. In: Han, W., et al. (eds.) Advances in Variational and Hemivariational Inequalities: Theory, Numerical Analysis, and Applications, Advances in Mechanics and Mathematics Series, vol. 33, pp. 355-380. Springer, Cham (2015)

19. Sofonea, M., Migórski, S.: Variational-Hemivariational Inequalities with Applications. Monographs and Research Notes in Mathematics. CRC, Boca Raton (2018)

20. Denkowski, Z., Migórski, S., Ochal, A.: A class of optimal control problems for piezoelectric frictional contact models. Nonlinear Anal. RWA 12, 1883-1895 (2011)

21. Matei, A., Micu, S.: Boundary optimal control for a frictional contact problem with normal compliance. Appl. Math. Optim. 78, 379-401 (2018)

22. Matei, A., Micu, S.: Boundary optimal control for nonlinear antiplane problems. Nonlinear Anal. 74, 1641-1652 (2011)

23. Migórski, S.: Evolution hemivariational inequalities in infinite dimension and their control. Nonlinear Anal. 47, 101-112 (2001)

24. Sofonea, M.: Optimal control of a class of variational-hemivariational inequalities in reflexive Banach spaces. Appl. Math. Optim. 79, 621-646 (2019). https://doi.org/10.1007/s00245-017-9450-0

25. Sofonea, M., Xiao, Y.B.: Boundary optimal control of a nonsmooth frictionless contact problem. Comput. Math. Appl. 78, 152-165 (2019)

26. Touzaline, A.: Optimal control of a frictional contact problem. Acta Math. Appl. Sin. Engl. Ser. 31, 991-1000 (2015)

27. Xiao, Y.B., Sofonea, M.: On the optimal control of variational-hemivariational inequalities. J. Math. Anal. Appl. 475, 364-384 (2019)

28. Gasinski, L., Migórski, S., Ochal, A.: Existence results for evolutionary inclusions and variationalhemivariational inequalities. Appl. Anal. 94, 1670-1694 (2014)

29. Sofonea, M., Migórski, S., Ochal, A.: Evolutionary inclusions and hemivariational inequalities. In: Han, W., et al. (eds.) Advances in Variational and Hemivariational Inequalities: Theory, Numerical 
Analysis, and Applications, Advances in Mechanics and Mathematics Series, vol. 33, pp. 39-64. Springer, Cham (2015)

30. Han, E., Migórski, S., Sofonea, M.: Analysis of a general dynamic history-dependent variationalhemivariational inequality. Nonlinear Anal. RWA 36, 69-88 (2017)

31. Migórski, S., Ochal, A., Sofonea, M.: Nonlinear Inclusions and Hemivariational Inequalities. Models and Analysis of Contact Problems, Advances in Mechanics and Mathematics Series, vol. 26. Springer, New York (2013)

32. Migórski, S., Ogorzaly, J.: A class of evolution variational inequalities with memory and its application to viscoelastic frictional contact problems. J. Math. Anal. Appl. 442, 685-702 (2016)

33. Migórski, S., Ogorzaly, J.: Dynamic history-dependent variational-hemivariational inequalities with applications to contact mechanics. Zeitschrift für angewandte Mathematik und Physik 68(1), 15 (2017). https://doi.org/10.1007/s00033-016-0758-4

34. Denkowski, Z., Migórski, S., Papageorgiou, N.S.: An Introduction to Nonlinear Analysis: Theory. Kluwer Academic, Dordrecht (2003)

35. Clarke, F.H.: Optimization and Nonsmooth Analysis. Wiley Interscience, New York (1983)

36. Denkowski, Z., Migórski, S., Papageorgiou, N.S.: An Introduction to Nonlinear Analysis: Applications. Kluwer Academic, Dordrecht (2003)

37. Kulig, A., Migórski, S.: Solvability and continuous dependence results for second order nonlinear inclusion with Volterra-type operator. Nonlinear Anal. 75, 4729-4746 (2012)

38. Sofonea, M., Matei, A.: Mathematical Models in Contact Mechanics, London Mathematical Society Lecture Note Series, vol. 398. Cambridge University Press, Cambridge (2012)

39. Zeng, B., Migórski, S.: Evolutionary subgradient inclusions with nonlinear weakly continuous operators and applications. Comput. Math. Appl. 75, 89-104 (2018)

40. Aubin, J.P., Cellina, A.: Differential Inclusions: Set-Valued Maps and Viability Theory. Springer, Berlin (1984)

41. Migórski, S., Ochal, A.: Quasi-static hemivariational inequality via vanishing acceleration approach. SIAM J. Math. Anal. 41, 1415-1435 (2009)

42. Peng, Z.: Existence and regularity results for doubly nonlinear inclusions with nonmonotone perturbation. Nonlinear Anal. 115, 71-88 (2015)

43. Balder, E.J.: Necessary and sufficient conditions for $L^{1}$-strong-weak lower semicontinuity of integral functionals. Nonlinear Anal. Theory Methods Appl. 11, 1399-1404 (1987)

44. Mordukhovich, B.S.: Variational Analysis and Generalized Differentiations, II: Applications. Springer, Berlin (2006)

45. Colombo, G., Henrion, R., Hoang, N.D., Mordukhovich, B.S.: Optimal control of the sweeping process over polyhedral controlled sets. J. Differ. Equ. 260, 3397-3447 (2016)

46. Colombo, G., Mordukhovich, B.S., Nguyen, D.: Optimal control of sweeping processes in robotics and traffic flow models. J. Optim. Theory Appl. 182, 439-472 (2019)

47. Hoang, N.D., Mordukhovich, B.S.: Extended Euler-Lagrange and Hamiltonian formalisms in optimal control of sweeping processes with controlled sweeping sets. J. Optim. Theory Appl. 180, 256-289 (2019)

Publisher's Note Springer Nature remains neutral with regard to jurisdictional claims in published maps and institutional affiliations. 